\title{
ALMOST MONOTONICITY FORMULAS FOR ELLIPTIC AND PARABOLIC OPERATORS WITH VARIABLE COEFFICIENTS
}

\author{
NORAYR MATEVOSYAN AND ARSHAK PETROSYAN
}

\begin{abstract}
In this paper we extend the results of Caffarelli-Jerison-Kenig [Ann. of Math. (2) 155 (2002)] and Caffarelli-Kenig [Amer. J. Math. 120 (1998)] by establishing an almost monotonicity estimate for pairs of continuous functions satisfying$$
u_{ \pm} \geq 0, \quad \mathcal{L} u_{ \pm} \geq-1, \quad u_{+} \cdot u_{-}=0
$$

in an infinite strip (global version) or a finite parabolic cylinder (localized version), where $\mathcal{L}$ is a uniformly parabolic operator

$$
\mathcal{L} u=\mathcal{L}_{\mathcal{A}, b, c} u:=\operatorname{div}(\mathcal{A}(x, s) \nabla u)+b(x, s) \cdot \nabla u+c(x, s) u-\partial_{s} u
$$

with double Dini continuous $\mathcal{A}$ and uniformly bounded $b$ and $c$. We also prove the elliptic counterpart of this estimate.

This closes the gap between the known conditions in the literature (both in the elliptic and parabolic case) imposed on $u_{ \pm}$in order to obtain an almost monotonicity estimate.

At the end of the paper, we demonstrate how to use this new almost monotonicity formula to prove the optimal $C^{1,1}$ regularity in a fairly general class of quasilinear obstacle-type free boundary problems.
\end{abstract}

\section{INTRODUCTION}

\subsection{Background.}

1.1.1. Original Monotonicity Formulas. In a seminal paper [ACF84], Alt, Caffarelli, and Friedman have proved the following monotonicity formula: if $u_{ \pm}$are two continuous functions in the unit ball $B_{1}$ in $\mathbb{R}^{n}$ such that

$$
u_{ \pm} \geq 0, \quad \Delta u_{ \pm} \geq 0, \quad u_{+} \cdot u_{-}=0 \quad \text { in } \quad B_{1}
$$

then the functional

$$
\varphi(r)=\varphi\left(r, u_{+}, u_{-}\right)=\frac{1}{r^{4}} \int_{B_{r}} \frac{\left|\nabla u_{+}\right|^{2}}{|x|^{n-2}} d x \int_{B_{r}} \frac{\left|\nabla u_{-}\right|^{2}}{|x|^{n-2}} d x
$$

is monotone nondecreasing in $r \in(0,1]$. This formula has been of fundamental importance in the regularity theory of free boundaries, especially in problems with

2000 Mathematics Subject Classification. Primary 35R35. Secondary 35K10, 35J25.

Key words and phrases. Monotonicity formulas, almost monotonicity formulas, elliptic ad parabolic equations, variable coefficients, divergence form, eigenvalue inequalities, Gaussian spaces, quasilinear obstacle type problems, type II superconductors.

N. Matevosyan is partially supported by the WWTF (Wiener Wissenschafts, Forschungs und Technologiefonds) project No. CI06 003 and by award No. KUK-I1-007-43, made by King Abdullah University of Science and Technology (KAUST).

A. Petrosyan is supported in part by NSF grant DMS-0701015. 
two phases. One of its applications is the ability to produce estimates of the type

$$
c_{n}\left|\nabla u_{+}(0)\right|^{2}\left|\nabla u_{-}(0)\right|^{2} \leq \varphi(0+) \leq \varphi(1 / 2) \leq C_{n}\left\|u_{+}\right\|_{L^{2}\left(B_{1}\right)}^{2}\left\|u_{-}\right\|_{L^{2}\left(B_{1}\right)}^{2},
$$

which are crucial in establishing the optimal regularity in a number of free boundary problems.

The parabolic counterpart of the monotonicity formula above has been established by Caffarelli [Caf93]: if $u_{ \pm}(x, s)$ are two continuous functions in the unit strip $S_{1}=\mathbb{R}^{n} \times(-1,0]$ with moderate growth at infinity and such that

$$
u_{ \pm} \geq 0, \quad\left(\Delta-\partial_{s}\right) u_{ \pm} \geq 0, \quad u_{+} \cdot u_{-}=0 \quad \text { in } \quad S_{1}
$$

then the functional

$$
\Phi\left(r, u_{+}, u_{-}\right)=\frac{1}{r^{4}} \int_{-r^{2}}^{0} \int_{\mathbb{R}^{n}}\left|\nabla u_{+}\right|^{2} G(x,-s) d x d s \int_{-r^{2}}^{0} \int_{\mathbb{R}^{n}}\left|\nabla u_{-}\right|^{2} G(x,-s) d x d s
$$

is monotone nondecreasing. (Here $G(x, t)$ is the heat kernel; see more on notations in Section 2.1.) Already [Caf93] contains a localized version of this monotonicity formula. It says that if $u_{ \pm}$are defined only in a parabolic cylinder $Q_{1}^{-}=B_{1} \times(-1,0]$ then for any spatial cutoff function $\psi \in C_{0}^{\infty}\left(B_{1}\right)$ such that $0 \leq \psi \leq 1,\left.\psi\right|_{B_{1 / 2}}=1$, the functional $\Phi(r)=\Phi\left(r, u_{+} \psi, u_{-} \psi\right)$ is almost monotone in a sense that

$$
\Phi(0+)-\Phi(r) \leq C e^{-c / r^{2}}\left\|u_{+}\right\|_{L^{2}\left(Q_{1}^{-}\right)}^{2}\left\|u_{-}\right\|_{L^{2}\left(Q_{1}^{-}\right)}^{2} .
$$

1.1.2. Almost Monotonicity Formulas. In recent years, several generalizations of the monotonicity formulas above has been obtained, mainly motivated by increasing the range of their applicability. These results share the same general trait: while relaxing conditions on $u_{ \pm}$, they give up the full monotonicity of $\varphi$ (or $\left.\Phi\right)$, but retain an estimate of the type (in the elliptic case)

$$
\varphi(0+) \leq C\left(\left\|u_{+}\right\|_{L^{2}\left(B_{1}\right)},\left\|u_{-}\right\|_{L^{2}\left(B_{1}\right)}\right),
$$

which still has a strong potential in applications. We call such results almost monotonicity formulas, even though, in fact, no monotonicity is left at all.

One notable result is the almost monotonicity formula of Caffarelli and Kenig [CK98] which generalizes the parabolic monotonicity formula of Caffarelli [Caf93] to the variable coefficient case: if $\mathcal{L}$ is a uniformly parabolic operator

$$
\mathcal{L} u=\mathcal{L}_{\mathcal{A}, b, c} u:=\operatorname{div}(\mathcal{A}(x, s) \nabla u)+b(x, s) \cdot \nabla u+c(x, s) u-\partial_{s} u
$$

with Dini continuous coefficient matrix $\mathcal{A}(x, s)$ and uniformly bounded $b(x, s)$ and $c(x, s)$, then for a pair of continuous functions $u_{ \pm}$in $Q_{1}^{-}=B_{1} \times(-1,0]$ satisfying

$$
u_{ \pm} \geq 0, \quad \mathcal{L} u_{ \pm} \geq 0, \quad u_{+} \cdot u_{-}=0 \quad \text { in } \quad Q_{1}^{-},
$$

we have an estimate

$$
\Phi\left(r, u_{+} \psi, u_{-} \psi\right) \leq C\left(\left\|u_{+}\right\|_{L^{2}\left(Q_{1}^{-}\right)}^{2}+\left\|u_{-}\right\|_{L^{2}\left(Q_{1}^{-}\right)}^{2}\right)^{2}
$$

for sufficiently small $r$, where $\psi \in C_{0}^{\infty}\left(B_{1}\right)$ is a cutoff function with $\left.\psi\right|_{B_{1 / 2}}=1$. A similar result can be proved also in the elliptic case.

Another notable result is the following estimate of Caffarelli, Jerison, and Kenig [CJK02]: if $u_{ \pm}$are continuous functions in the unit ball, satisfying

$$
u_{ \pm} \geq 0, \quad \Delta u_{ \pm} \geq-1, \quad u_{+} \cdot u_{-}=0 \quad \text { in } B_{1},
$$


then we have

$$
\varphi\left(r, u_{+}, u_{-}\right) \leq C\left(1+\left\|u_{+}\right\|_{L^{2}\left(B_{1}\right)}^{2}+\left\|u_{-}\right\|_{L^{2}\left(B_{1}\right)}^{2}\right)^{2}
$$

for $r \in(0,1 / 2)$. Even though one only changes the condition $\Delta u_{ \pm} \geq 0$ to $\Delta u_{ \pm} \geq$ -1 compared to the original monotonicity formula of [ACF84], the proof of this estimate is very involved and is based on a sophisticated iteration scheme. Recently, a parabolic analogue of this result has been proved by Edquist and Petrosyan [EP08] for a pair of functions satisfying

$$
u_{ \pm} \geq 0, \quad\left(\Delta-\partial_{s}\right) u_{ \pm} \geq-1, \quad u_{+} \cdot u_{-}=0 \quad \text { in } \quad S_{1} .
$$

A localized version of this formula for functions $u_{ \pm}$defined only in $Q_{1}^{-}$has also been proved.

1.2. Main Results. The main purpose of this paper is to further extend the almost monotonicity formulas above, to the pairs of functions satisfying

$$
u_{ \pm} \geq 0, \quad \mathcal{L} u \geq-1, \quad u_{+} \cdot u_{-}=0 \quad \text { in } \quad S_{1},
$$

where $\mathcal{L}$ is a uniformly parabolic operator with variable coefficients (Theorem I). Essentially, we accomplish this by combining the techniques from aforementioned papers [CK98, CJK02,EP08]. We further prove the localized version of this estimate (Theorem II), as well as its elliptic counterpart (Theorem III).

To be more specific, let $\mathcal{L}$ be a uniformly parabolic operator in $S_{1}:=\mathbb{R}^{n} \times(-1,0]$

$$
\mathcal{L} u=\mathcal{L}_{\mathcal{A}, b, c} u:=\operatorname{div}(\mathcal{A}(x, s) \nabla u)+b(x, s) \cdot \nabla u+c(x, s) u-\partial_{s} u
$$

with the following assumptions on the coefficients: there exist positive constants $\lambda$, $\mu$, and a modulus of continuity $\omega(\rho)$ such that at every $(x, s) \in S_{1}$ we have

$$
\begin{gathered}
\lambda|\xi|^{2} \leq \xi \cdot \mathcal{A}(x, s) \xi \leq \frac{1}{\lambda}|\xi|^{2} \quad \text { for any } \xi \in \mathbb{R}^{n} \\
\|\mathcal{A}(x, s)-\mathcal{A}(0,0)\| \leq \omega\left(\left(|x|^{2}+|s|\right)^{1 / 2}\right) \\
|b(x, s)|+|c(x, s)| \leq \mu
\end{gathered}
$$

Throughout the paper we will assume that the modulus of continuity $\omega(\rho)$ satisfies the double Dini condition

$$
\int_{0}^{1} \frac{1}{r} \int_{0}^{r} \frac{\omega(\rho)}{\rho} d \rho d r=\int_{0}^{1} \frac{\omega(\rho) \log \frac{1}{\rho}}{\rho} d \rho<\infty .
$$

Note that a Hölder modulus of continuity $\omega(r)=C r^{\alpha}, 0<\alpha<1$, readily satisfies (1.5). In fact, a somewhat weaker condition

$$
\int_{0}^{1} \frac{1}{r}\left(\int_{0}^{r} \frac{\omega(\rho)^{2}}{\rho} d \rho\right)^{1 / 2} d r<\infty
$$

would be sufficient for us, see Remark after Proposition 3.1. Note that $\left(1.5^{\prime}\right)$ follows readily from $(1.5)$, since

$$
2\left(\int_{0}^{r} \frac{\omega(\rho)^{2}}{\rho} d \rho\right)^{1 / 2} \leq 2\left(\omega(r) \int_{0}^{r} \frac{\omega(\rho)}{\rho} d \rho\right)^{1 / 2} \leq \omega(r)+\int_{0}^{r} \frac{\omega(\rho)}{\rho} d \rho .
$$

Likewise, let $\ell$ be a uniformly elliptic operator in $B_{1}$

$$
\ell u=\ell_{\mathcal{A}, b, c} u:=\operatorname{div}(\mathcal{A}(x) \nabla u)+b(x) \cdot \nabla u+c(x) u
$$


such that

$$
\begin{gathered}
\lambda|\xi|^{2} \leq \xi \cdot \mathcal{A}(x) \xi \leq \frac{1}{\lambda}|\xi|^{2} \quad \text { for any } \xi \in \mathbb{R}^{n} \\
\|\mathcal{A}(x)-\mathcal{A}(0)\| \leq \omega(|x|) \\
|b(x)|+|c(x)| \leq \mu
\end{gathered}
$$

for positive constants $\lambda$ and $\mu$ and a modulus of continuity $\omega(\rho)$ satisfying (1.5).

The main results of this paper are the following three theorems. We refer to Section 2.1 for the notation used in the statement of these results.

Theorem I (Parabolic Almost Monotonicity Formula). Suppose we have two continuous functions $u_{ \pm}(x, s)$ in the unit strip $S_{1}$ that are also in $V_{\mathrm{loc}}^{2}\left(S_{1}\right)$ and satisfy

$$
u_{ \pm} \geq 0, \quad \mathcal{L} u_{ \pm} \geq-1, \quad u_{+} \cdot u_{-}=0 \quad \text { in } \quad S_{1}
$$

with operator (1.1) having the properties (1.2)-(1.5). Assume also that $u_{ \pm}$have moderate growth at infinity, so that

$$
M_{ \pm}^{2}:=\iint_{S_{1}} u_{ \pm}(x, s)^{2} e^{-x^{2} / 32} d x d s<\infty .
$$

Then the functional

$$
\Phi(r):=r^{-4} A_{+}(r) A_{-}(r), \quad \text { where } \quad A_{ \pm}(r):=\iint_{S_{r}}\left|\nabla u_{ \pm}\right|^{2} d \gamma
$$

satisfies

$$
\Phi(r) \leq C_{\omega}\left(1+M_{+}^{2}+M_{-}^{2}\right)^{2}, \quad \text { for } 0<r \leq r_{\omega} .
$$

Next we state the localized version of Theorem I.

Theorem II (Localized Parabolic Almost Monotonicity Formula). Suppose we have two continuous functions $u_{ \pm}(x, s)$ in the parabolic halfcylinder $Q_{1}^{-}$that are also in $V^{2}\left(Q_{1}^{-}\right)$and satisfy

$$
u_{ \pm} \geq 0, \quad \mathcal{L} u_{ \pm} \geq-1, \quad u_{+} \cdot u_{-}=0 \quad \text { in } \quad Q_{1}^{-}
$$

with operator (1.1) having the properties (1.2)-(1.5). Let $\psi \in C_{0}^{\infty}\left(B_{1}\right)$ be a cutoff function such that

$$
\left.\psi\right|_{B_{1 / 2}}=1, \quad \operatorname{supp} \psi \subset B_{3 / 4} .
$$

Define $w_{ \pm}(x, s)=u_{ \pm}(x, s) \psi(x)$. Then the functional

$$
\Phi(r):=r^{-4} A_{+}(r) A_{-}(r), \quad \text { where } A_{ \pm}(r):=\iint_{S_{r}}\left|\nabla w_{ \pm}\right|^{2} d \gamma,
$$

satisfies

$$
\Phi(r) \leq C_{\omega, \psi}\left(1+\left\|u_{+}\right\|_{L^{2}\left(Q_{1}^{-}\right)}^{2}+\left\|u_{-}\right\|_{L^{2}\left(Q_{1}^{-}\right)}^{2}\right)^{2}, \quad \text { for } 0<r \leq r_{\omega} .
$$

Finally, we state the result in the elliptic case.

Theorem III (Elliptic Almost Monotonicity Formula). Suppose we have two continuous functions $u_{ \pm}(x, s)$ in the unit ball $B_{1}$ that satisfy

$$
u_{ \pm} \geq 0, \quad \ell u_{ \pm} \geq-1, \quad u_{+} \cdot u_{-}=0 \quad \text { in } \quad B_{1}
$$

with operator (1.6) having the properties (1.7)-(1.9). Then the functional

$$
\varphi(r):=r^{-4} a_{+}(r) a_{-}(r), \quad \text { where } a_{ \pm}(r):=\int_{B_{r}} \frac{\left|\nabla u_{ \pm}\right|^{2}}{|x|^{n-2}} d x
$$


satisfies

$$
\varphi(r) \leq C_{\omega}\left(1+\left\|u_{+}\right\|_{L^{2}\left(B_{1}\right)}^{2}+\left\|u_{-}\right\|_{L^{2}\left(B_{1}\right)}^{2}\right)^{2}, \quad \text { for } 0<r \leq r_{\omega} .
$$

\subsection{Structure of the Paper.}

- Section 2 contains notations as well preliminary results that will be used later in the paper.

- In Section 3 we prove Theorem I (global parabolic case). The technical core of the proof is Proposition 3.1, followed by Proposition 3.2-3.4, which allow to establish the iterative estimates in Proposition 3.5-3.6, ultimately implying Theorem I.

- In Section 4 (local parabolic case) we prove Theorem II. The proof is essentially the same, only we have to take into account new terms coming from the cutoff function, which turn out to be exponentially small.

- Section 5 establishes Theorem III (elliptic case) as a direct corollary of Theorem II.

- In Section 6, we give a variant of the almost monotonicity formula (in local parabolic case) under additional growth assumption near the origin.

- Finally, in Section 7 we give an application of the new almost monotonicity formulas (in the elliptic case) to a quasilinear obstacle type problem that arises in superconductivity.

\section{Notation And Preliminaries}

2.1. Notation. Throughout the paper we will use the following notations:

$$
\begin{array}{ll}
B_{r}(x)=\left\{y \in \mathbb{R}^{n}:|x-y|<r\right\} & \text { (spatial ball) } \\
B_{r}=B_{r}(0) & \text { (lower parabolic cylinder) } \\
Q_{r}^{-}=B_{r} \times\left(-r^{2}, 0\right] & \text { (infinite strip) } \\
S_{r}=\mathbb{R}^{n} \times\left(-r^{2}, 0\right] & x \in \mathbb{R}^{n}, t>0 \quad \text { (the heat kernel) } \\
G(x, t)=\frac{1}{(4 \pi t)^{n / 2}} e^{-|x|^{2} / 4 t}, & \\
d \gamma(x, s)=G(x,-s) d x d s & \\
d \gamma^{s}(x)=G(x,-s) d x & \text { (the standard Gaussian measure) } \\
d \nu=d \gamma^{-1 / 2} & \\
\Delta u=\sum_{i=1}^{n} \partial_{x_{i} x_{i}} u & \text { (spatial gradient) } \\
\nabla u=\nabla_{x} u=\left(\partial_{x_{1}} u, \ldots, \partial_{x_{n}} u\right)
\end{array}
$$

- For integrals in space and time we use the double-integral sign $\iint$, regardless of the space dimension, while for the integrals in space only we use the single-integral sign $\int$.

- By a modulus of continuity we understand a continuous nondecreasing function $\omega:(0, \infty) \rightarrow(0, \infty)$ such that $\omega(0+)=0$.

2.1.1. Convention for Constants. We will use the following convention for denoting various constants that appear in this paper, unless stated otherwise. 


$$
\begin{array}{ll}
c_{n}, C_{n} & \text { Dimensional constants, depending only on dimension } n \\
c, c_{0}, C, C_{0} & \text { Universal constants, depending only on the structural con- } \\
& \text { stants } \lambda, \mu \text { in }(1.2)-(1.4) \text { or }(1.7)-(1.9) \text { and dimension } n \\
c_{f}, C_{f} & \text { Constants depending also on } f \text { (a constant or a function) } \\
& \text { in addition to } \lambda, \mu, n \\
c_{f_{1}, \ldots, f_{k}}, C_{f_{1}, \ldots, f_{k}} & \text { Constants depending on } f_{1}, \ldots, f_{k} \text { (constants or functions) } \\
& \text { in addition to } \lambda, \mu, n .
\end{array}
$$

2.2. Notion of Solution. We use the notion of a weak solution of the inequality $\mathcal{L} u \geq f$ in the sense of [LSU68]. More specifically, let $\Omega$ be an open subset of $\mathbb{R}^{n}$, $T>0, \Omega_{T}=\Omega \times(-T, 0]$ and let

$$
V^{2}\left(\Omega_{T}\right):=C\left((-T, 0] ; L^{2}(\Omega)\right) \cap L^{2}\left((-T, 0] ; W^{1,2}(\Omega)\right)
$$

be the Banach space with the norm

$$
\|u\|_{V^{2}\left(\Omega_{T}\right)}^{2}=\sup _{-T<s \leq 0} \int_{\Omega} u(x, s)^{2} d x+\iint_{\Omega_{T}}|\nabla u|^{2} d x d s .
$$

The corresponding space $V_{0}^{2}\left(\Omega_{T}\right)$ is defined as the closure of $C_{0}^{\infty}\left(\Omega_{T}\right)$ in $V^{2}\left(\Omega_{T}\right)$. Further, we say $u \in V_{\text {loc }}^{2}\left(\Omega_{T}\right)$ if $u \in V^{2}\left(\Omega_{T^{\prime}}^{\prime}\right)$ for any $\Omega^{\prime} \Subset \Omega$ and $0<T^{\prime}<T$.

We say that $u \in V^{2}\left(\Omega_{T}\right)$ is a weak solution of $\mathcal{L} u \geq f$ with $f \in L^{2}\left(\Omega_{T}\right)$ if

$$
\iint_{\Omega_{T}}-\nabla \eta \cdot \mathcal{A} \nabla u+\eta(b \cdot \nabla u+c u)+\left(\partial_{s} \eta\right) u d x d s \geq \iint_{\Omega_{T}} f \eta d x d s
$$

for any $\eta \in V_{0}^{2}\left(\Omega_{T}\right)$ vanishing on $\Omega \times\{0\}$. This is equivalent to saying that $\mathcal{L} u \geq f$ in $\Omega \times(-T, 0)$ in the sense of distributions.

Lemma 2.1 (Energy Inequality). Let $u \in V^{2}\left(Q_{1}^{-}\right)$be a weak solution of $\mathcal{L} u \geq f$ in $Q_{1}^{-}$. Then

$$
\|u\|_{V^{2}\left(Q_{3 / 4}^{-}\right)}^{2} \leq C\left(\|u\|_{L^{2}\left(Q_{3 / 4}^{-}\right)}^{2}+\|f\|_{L^{2}\left(Q_{3 / 4}^{-}\right)}^{2}\right) .
$$

In the expanded form, the Energy Inequality reads as

$$
\sup _{-9 / 16<s \leq 0} \int_{B_{3 / 4}} u(x, s)^{2} d x+\iint_{Q_{3 / 4}^{-}}|\nabla u|^{2} d x d s \leq C \iint_{Q_{1}^{-}}\left(u^{2}+f^{2}\right) d x d s .
$$

We refer to [LSU68] for a proof.

2.3. Inequalities in Gaussian Spaces. In this section we collected some inequalities for functions in Gaussian spaces that we are going to use in this paper. Recall that $d \gamma^{s}=G(x,-s) d x$ for $s<0$. In particular $d \nu=d \gamma^{-1 / 2}$ is the standard Gaussian measure. We say $f \in W^{1,2}\left(\mathbb{R}^{n}, d \gamma^{s}\right)$ whenever $f \in L^{2}\left(\mathbb{R}^{n}, d \gamma^{s}\right)$ and all its distributional derivatives $\partial_{x_{i}} f \in L^{2}\left(\mathbb{R}^{n}, d \gamma^{s}\right), i=1, \ldots, n$.

Lemma 2.2 (Log-Sobolev Inequality). For any $u \in W^{1,2}\left(\mathbb{R}^{n}, d \gamma^{s}\right)$ we have

$$
\int_{\mathbb{R}^{n}} u^{2} \log u^{2} d \gamma^{s} \leq\left(\int_{\mathbb{R}^{n}} u^{2} d \gamma^{s}\right) \log \left(\int_{\mathbb{R}^{n}} u^{2} d \gamma^{s}\right)+4|s| \int_{\mathbb{R}^{n}}|\nabla u|^{2} d \gamma^{s} .
$$

For a proof, see [Gro75]. 
Lemma 2.3 (Poincaré Inequality in Gaussian Spaces). For any $v \in W^{1,2}\left(\mathbb{R}^{n}, d \gamma^{s}\right)$ with $\int_{\mathbb{R}^{n}} v d \gamma^{s}=0$ we have

$$
\int_{\mathbb{R}^{n}} v^{2} d \gamma^{s} \leq 2|s| \int_{\mathbb{R}^{n}}|\nabla v|^{2} d \gamma^{s} .
$$

For a proof of a generalization, see [Bec89].

Lemma 2.4 (Eigenvalue Inequality in Disjoint Domains). For a nonempty open set $\Omega \subset \mathbb{R}^{n}$ define

$$
\lambda^{s}(\Omega)=\inf \frac{\int_{\Omega}|\nabla f|^{2} d \gamma^{s}}{\int_{\Omega} f^{2} d \gamma^{s}},
$$

where the infimum is taken over all nonzero functions $f \in C^{0,1}\left(\mathbb{R}^{n}\right)$ vanishing on $\mathbb{R}^{n} \backslash \Omega$. Then for any two nonempty disjoints $\Omega_{ \pm}$be two nonempty disjoint open sets in $\mathbb{R}^{n}$, we have

$$
\lambda^{s}\left(\Omega_{+}\right)+\lambda^{s}\left(\Omega_{-}\right) \geq 2 \lambda^{s}\left(\mathbb{R}_{+}^{n}\right)=1 /|s| .
$$

This inequality is originally due to Beckner, Kenig, and Pipher [BKP98 $]^{1}$. It is closely related to the inequality of Friedland and Hayman [FH76] for eigenvalues of disjoint domains on the sphere.

\section{Parabolic Formula}

The purpose of this section is to prove Theorem I. The technical core of the proof is Proposition 3.1, followed by Propositions 3.2-3.4. The latter provide the technical background for establishing the iterative inequalities in Propositions 3.53.6 that ultimately imply Theorem I.

3.1. Initial Reduction. First, we make few simplifications.

$\left.1^{\circ}\right)$ Without loss of generality we may assume that

$$
\mathcal{A}(0,0)=I \text {. }
$$

Then the difference

$$
\mathcal{B}(x, s)=I-\mathcal{A}(x, s)
$$

will satisfy

$$
\|\mathcal{B}(x, s)\| \leq \omega\left(\left(|x|^{2}+|s|\right)^{1 / 2}\right)
$$

with a double Dini $\omega$ as in (1.5). Besides, we may also assume that for any $(x, s) \in$ $S_{1}$

$$
\|\mathcal{B}(x, s)\| \leq \frac{C_{n}}{\lambda} .
$$

$2^{\circ}$ ) Further, we may assume

$$
c(x, s)=0 .
$$

Indeed, if $u(x, s)$ satisfies

$$
u \geq 0, \quad \mathcal{L}_{\mathcal{A}, b, c} u \geq-1
$$

\footnotetext{
${ }^{1}$ Since [BKP98] is not published, we refer to Section 2.4 in [CK98] for the proof of (2.1).
} 
then

$$
\begin{aligned}
\mathcal{L}_{\mathcal{A}, b, 0}\left(e^{-\mu s} u(x, s)\right) & =e^{-\mu s}\left[\mathcal{L}_{\mathcal{A}, b, 0} u(x, s)+\mu u(x, s)\right] \\
& \geq e^{-\mu s} \mathcal{L}_{\mathcal{A}, b, c} u \geq-e^{-\mu s} \geq-1 .
\end{aligned}
$$

$\left.3^{\circ}\right)$ Finally, without loss of generality we may assume

$$
M_{ \pm} \leq 1
$$

and prove that

$$
\Phi\left(r, u_{+}, u_{-}\right) \leq C_{\omega}, \quad \text { for } r \leq r_{\omega} .
$$

In the general case, we may replace $u_{ \pm}$with $\tilde{u}_{ \pm}=u_{ \pm} /\left(1+M_{ \pm}\right)$and use that

$$
\Phi\left(r, u_{+}, u_{-}\right)=\Phi\left(r, \tilde{u}_{+}, \tilde{u}_{-}\right)\left(1+M_{+}\right)^{2}\left(1+M_{-}\right)^{2}
$$

to arrive at the conclusion of Theorem I.

\subsection{Main Estimates.}

Proposition 3.1 (cf. [EP08, Propositions 1.1 and 2.1]). Let $u \in C\left(S_{1}\right) \cap V_{\mathrm{loc}}^{2}\left(S_{1}\right)$ satisfy

$$
u \geq 0, \quad \mathcal{L}_{\mathcal{A}, b, 0} u \geq-1 \quad \text { in } S_{1}
$$

Suppose also

$$
\iint_{S_{1}} u(x, s)^{2} e^{-x^{2} / 32} d x d s \leq 1
$$

Then

$$
\begin{aligned}
\left(1-c_{n} \theta(r)\right) \iint_{S_{r}}|\nabla u|^{2} d \gamma & \leq C_{0} r^{4}+C_{n} r^{2}\left(\int_{\mathbb{R}^{n}} u\left(\cdot,-r^{2}\right)^{2} d \gamma^{-r^{2}}\right)^{1 / 2} \\
& +\frac{1}{2} \int_{\mathbb{R}^{n}} u\left(\cdot,-r^{2}\right)^{2} d \gamma^{-r^{2}}
\end{aligned}
$$

for any $0<r \leq r_{\omega}$, where

$$
\theta(r)=C r+\omega\left(r^{1 / 2}\right)+\left(\int_{0}^{r^{2}} \frac{\omega\left(\rho^{1 / 4}\right)^{2}}{\rho} d \rho\right)^{1 / 2} .
$$

Remark. The double Dini condition (1.5) on $\omega(r)$ is to ensures that $\theta(r)$ above satisfies the Dini condition $\int_{0}^{1}(\theta(r) / r) d r<\infty$. Indeed, recall that (1.5) implies $\left(1.5^{\prime}\right)$ and notice that the Dini integrability of any function $\sigma(r)$ is equivalent to that of $\sigma\left(r^{\alpha}\right)$ for any $\alpha>0$, since $\int_{0}^{1}(\sigma(r) / r) d r=\alpha \int_{0}^{1}\left(\sigma\left(r^{\alpha}\right) / r\right) d r$.

Also note that in fact the term $\omega\left(r^{1 / 2}\right)$ is superfluous in the formula for $\theta(r)$, in the sense that $\theta(r)$ can be replaced with

$$
\vartheta(r)=C r+\left(\int_{0}^{r^{2}} \frac{\omega\left(\rho^{1 / 4}\right)^{2}}{\rho} d \rho\right)^{1 / 2} .
$$

Indeed, modifying slightly the proof (splitting $\int_{\mathbb{R}^{n}}=\int_{|x| \leq \frac{1}{2}|s|^{1 / 4}}+\int_{|x| \geq \frac{1}{2}|s|^{1 / 4}}$ ) one may replace $\theta(r)$ by $\theta(r / 4)$, which is easily majorized by $C_{n} \vartheta(r)$. 
Proof of Proposition 3.1. Because of the assumption $\mathcal{A}(0,0)=I$, we view $\mathcal{L}$ as a perturbation of the heat operator $\Delta-\partial_{s}$ and write

$$
\begin{aligned}
\left(\Delta-\partial_{s}\right) u & =\mathcal{L}_{\mathcal{A}, b, 0} u+\operatorname{div}(\mathcal{B}(x, s) \nabla u)-b(x, s) \cdot \nabla u, \\
\left(\Delta-\partial_{s}\right)\left(u^{2} / 2\right) & =u\left(\mathcal{L}_{\mathcal{A}, b, 0} u+\operatorname{div}(\mathcal{B} \nabla u)-b \nabla u\right)+|\nabla u|^{2} \\
& \geq-u+u \operatorname{div}(\mathcal{B} \nabla u)-u b \nabla u+|\nabla u|^{2},
\end{aligned}
$$

in the sense of distributions. Thus, the latter inequality means that for any nonnegative $\eta \in C_{0}^{\infty}\left(S_{1}\right)$ and $-1<s_{2}<s_{1} \leq 0$ we have

$$
\begin{array}{r}
\frac{1}{2} \int_{s_{2}}^{s_{1}} \int_{\mathbb{R}^{n}} u^{2}\left(\Delta+\partial_{s}\right) \eta d x d s-\frac{1}{2} \int_{\mathbb{R}^{n}}\left[u^{2}\left(\cdot, s_{1}\right) \eta\left(\cdot, s_{1}\right)-u^{2}\left(\cdot, s_{2}\right) \eta\left(\cdot, s_{2}\right)\right] d x \\
\geq \int_{s_{2}}^{s_{1}} \int_{\mathbb{R}^{n}}\left[-u \eta-\nabla(u \eta) \mathcal{B} \nabla u-\eta u b \nabla u+\eta|\nabla u|^{2}\right] d x d s .
\end{array}
$$

Note also that one can actually take $\eta(x, s)=G(x,-s)$ in the formula above, because of the growth assumption that we impose on $u$, as well as the energy inequality (Lemma 2.1). The same argument justifies also the formal integration by parts in space variables that we are going to use throughout the proof.

Now, the inequality (3.7) implies that

$$
\begin{aligned}
\iint_{S_{r}}|\nabla u|^{2} d \gamma \leq & \frac{1}{2} \iint_{S_{r}}\left(\Delta-\partial_{s}\right)\left(u^{2}\right) d \gamma+\iint_{S_{r}} u d \gamma \\
& -\iint_{S_{r}} u \operatorname{div}(\mathcal{B} \nabla u) d \gamma-\iint_{S_{r}} u b \nabla u d \gamma \\
= & : \frac{1}{2} I_{1}+I_{2}+I_{3}+I_{4} .
\end{aligned}
$$

The rest of the proof consists in a careful estimation of each of the integrals $I_{1}, I_{2}$, $I_{3}, I_{4}$.

Before starting the estimations, we introduce the following convention for the modulus of continuity $\theta(r)$ : we let

$$
\theta(r)=\theta_{C}(r):=C r+\omega\left(r^{1 / 2}\right)+\left(\int_{0}^{r^{2}} \frac{\omega\left(\rho^{1 / 4}\right)^{2}}{\rho} d \rho\right)^{1 / 2},
$$

where $C>0$ is a generic universal constant, that may change due course. This convention allows to use inequalities of the type

$$
\theta^{2}(r) \leq \theta(r), \quad C r+\theta(r) \leq \theta(r), \quad \ldots
$$

for $r<r_{\omega}$. Thus, the first inequality assumes that $\theta(r) \leq 1$ for $r<r_{\omega}$, while the second one should be understood in the sense that $C r+\theta_{C_{1}}(r) \leq \theta_{C_{2}}(r)$.

$\left.1^{\circ}\right)$ To estimate $I_{1}$, we integrate by parts and use that $\left(\Delta+\partial_{s}\right) G(x,-s)=0$ in $\{s<0\}$

$$
\begin{aligned}
I_{1} & =-\iint_{S_{r}} \partial_{s}\left(u^{2} G(x,-s)\right) d x d s=\int_{\mathbb{R}^{n}} u\left(\cdot,-r^{2}\right)^{2} d \gamma^{-r^{2}}-u(0,0)^{2} \\
& \leq \int_{\mathbb{R}^{n}} u\left(\cdot,-r^{2}\right)^{2} d \gamma^{-r^{2}} .
\end{aligned}
$$


$2^{\circ}$ ) To estimate $I_{2}$, consider the weighted averages of $u(\cdot, s)$

$$
m(s)=\int_{\mathbb{R}^{n}} u(\cdot, s) d \gamma^{s}=\int_{\mathbb{R}^{n}} u(x, s) G(x,-s) d x .
$$

Then, for $-r^{2} \leq s_{2} \leq s_{1} \leq 0$, using (3.6) and the equality $\left(\Delta+\partial_{s}\right) G(x,-s)=0$ in $\{s<0\}$, we have

$$
\begin{aligned}
m\left(s_{1}\right)-m\left(s_{2}\right) & =-\int_{s_{2}}^{s_{1}} \int_{\mathbb{R}^{n}}\left(\Delta-\partial_{s}\right) u G(x,-s) d x d s \\
& \leq \int_{s_{2}}^{s_{1}} \int_{\mathbb{R}^{n}}(1-\operatorname{div}(\mathcal{B} \nabla u)+b \nabla u) G(x,-s) d x d s \\
& =\left(s_{1}-s_{2}\right)+\int_{s_{2}}^{s_{1}} \int_{\mathbb{R}^{n}}(\mathcal{B} \nabla u \nabla G(x,-s)+b \nabla u G(x,-s)) d x d s \\
& =\left(s_{1}-s_{2}\right)+\int_{s_{2}}^{s_{1}}\left(E_{2}(s)+H_{2}(s)\right) d s,
\end{aligned}
$$

where

$$
E_{2}(s)=\int_{\mathbb{R}^{n}} \mathcal{B} \nabla u \nabla G(x,-s) d x, \quad H_{2}(s)=\int_{\mathbb{R}^{n}} b \nabla u G(x,-s) d x .
$$

We next estimate $E_{2}(s)$ and $H_{2}(s)$.

2. $\left.i^{\circ}\right)$ To estimate $E_{2}(s)$, split it into two parts

$$
E_{2}(s)=\int_{|x| \geq|s|^{1 / 4}}+\int_{|x| \leq|s|^{1 / 4}}=: E_{21}(s)+E_{22}(s) .
$$

Using the equality $\nabla G(x,-s)=\frac{x}{2 s} G(x,-s)$ and applying the Cauchy-Schwarz inequality, we obtain

$$
\begin{aligned}
\left|E_{21}(s)\right| & =\left|\int_{|x| \geq|s|^{1 / 4}} \mathcal{B} \nabla u \nabla G(x,-s)\right| \leq C \int_{|x| \geq|s|^{1 / 4}}|\nabla u| \frac{|x|}{2|s|} G(x,-s) d x \\
& \leq C\left(\int_{\mathbb{R}^{n}}|\nabla u|^{2} d \gamma^{s}\right)^{1 / 2}\left(\int_{|x| \geq|s|^{1 / 4}} \frac{|x|^{2}}{4|s|^{2}} G(x,-s) d x\right)^{1 / 2} \\
& \leq C e^{-c_{0} /|s|^{1 / 2}}\left(\int_{\mathbb{R}^{n}}|\nabla u|^{2} d \gamma^{s}\right)^{1 / 2},
\end{aligned}
$$

where we have used that $\int_{|x| \geq|s|^{1 / 4}} \frac{|x|^{2}}{4|s|^{2}} G(x,-s) d x \leq C_{n} e^{-2 c_{0} /|s|^{1 / 2}}$.

Further,

$$
\begin{aligned}
\left|E_{22}(s)\right| & =\left|\int_{|x| \leq|s|^{1 / 4}} \mathcal{B} \nabla u \nabla G(x,-s)\right| \leq \omega\left(|s|^{1 / 4}\right) \int_{\mathbb{R}^{n}}|\nabla u| \frac{|x|}{2|s|} G(x,-s) \\
& \leq \omega\left(|s|^{1 / 4}\right)\left(\int_{\mathbb{R}^{n}}|\nabla u|^{2} d \gamma^{s}\right)^{1 / 2}\left(\int_{\mathbb{R}^{n}} \frac{|x|^{2}}{4|s|^{2}} G(x,-s)\right)^{1 / 2} .
\end{aligned}
$$

Now, note that

$$
\int_{\mathbb{R}^{n}} \frac{|x|^{2}}{|s|} G(x,-s) d x=-2 \int_{\mathbb{R}^{n}} x \cdot \nabla G(x,-s)=2 \int_{\mathbb{R}^{n}}(\operatorname{div} x) G=2 n
$$


Therefore,

$$
\left|E_{22}(s)\right| \leq C_{n} \frac{\omega\left(|s|^{1 / 4}\right)}{|s|^{1 / 2}}\left(\int_{\mathbb{R}^{n}}|\nabla u|^{2} d \gamma^{s}\right)^{1 / 2} .
$$

Thus, we obtain that

$$
\left|E_{2}(s)\right| \leq C_{n}\left(C+\frac{\omega\left(|s|^{1 / 4}\right)}{|s|^{1 / 2}}\right)\left(\int_{\mathbb{R}^{n}}|\nabla u|^{2} d \gamma^{s}\right)^{1 / 2} .
$$

For $\mathrm{H}_{2}(s)$, we obtain

$$
\left|H_{2}(s)\right| \leq \mu \int_{\mathbb{R}^{n}}|\nabla u| G(x,-s) \leq C\left(\int_{\mathbb{R}^{n}}|\nabla u|^{2} d \gamma^{s}\right)^{1 / 2} .
$$

2. $\left.i i^{\circ}\right)$ We continue the estimation of $m(s)$. Integrating the estimates for $\left|E_{2}(s)\right|$ and $\left|H_{2}(s)\right|$ in $2 . \mathrm{i}^{\circ}$ and applying the Cauchy-Schwarz inequality, we obtain for $-r^{2} \leq s \leq 0$

$$
\begin{aligned}
m(s) & \leq m\left(-r^{2}\right)+r^{2}+C_{n} \int_{-r^{2}}^{0}\left(C+\frac{\omega\left(|s|^{1 / 4}\right)}{|s|^{1 / 2}}\right)\left(\int_{\mathbb{R}^{n}}|\nabla u|^{2} d \gamma^{s}\right)^{1 / 2} d s \\
& \leq m\left(-r^{2}\right)+r^{2}+C_{n} \theta(r)\left(\iint_{S_{r}}|\nabla u|^{2} d \gamma\right)^{1 / 2} .
\end{aligned}
$$

Integrating one more time gives

$$
\begin{aligned}
I_{2}=\iint_{S_{r}} u d \gamma & =\int_{-r^{2}}^{0} m(s) d s \leq r^{2} m\left(-r^{2}\right)+r^{4}+C_{n} r^{2} \theta(r)\left(\iint_{S_{r}}|\nabla u|^{2} d \gamma\right)^{1 / 2} \\
& \leq r^{2} m\left(-r^{2}\right)+r^{4}+C_{n} r^{4}+\theta(r)^{2} \iint_{S_{r}}|\nabla u|^{2} d \gamma \\
& \leq r^{2} m\left(-r^{2}\right)+C_{n} r^{4}+\theta(r) \iint_{S_{r}}|\nabla u|^{2} d \gamma
\end{aligned}
$$

That is,

$$
I_{2} \leq r^{2} m\left(-r^{2}\right)+C_{n} r^{4}+\theta(r) \iint_{S_{r}}|\nabla u|^{2} d \gamma
$$

$3^{\circ}$ ) We now estimate

$$
I_{3}=-\iint_{S_{r}} u \operatorname{div}(\mathcal{B} \nabla u) d \gamma=\iint_{S_{r}} \nabla u \mathcal{B} \nabla u G+\iint_{S_{r}} u \mathcal{B} \nabla u \nabla G=: I_{31}+I_{32} .
$$

$\left.3 . i^{\circ}\right)$ Repeating the arguments as before, we obtain

$$
\begin{aligned}
\left|I_{31}\right| & \leq \iint_{|x| \leq|s|^{1 / 4}} \omega\left(|s|^{1 / 4}\right)|\nabla u|^{2} G(x,-s)+C \iint_{|x| \geq|s|^{1 / 4}}|\nabla u|^{2} G \\
& \leq \omega\left(r^{1 / 2}\right) \iint_{S_{r}}|\nabla u|^{2} d \gamma+C e^{-c_{0} / r},
\end{aligned}
$$

where in the estimation of the second integral over we have used that

$$
G(x,-s) \leq e^{-c_{0} /|s|^{1 / 2}} e^{-x^{2} / 8}, \quad \text { for }|x| \geq|s|^{1 / 4}
$$

and the following corollary from the Energy Inequality (Lemma 2.1)

$$
\iint_{S_{r}}|\nabla u|^{2} e^{-x^{2} / 8} d x d s \leq C \iint_{S_{1}} u^{2} e^{-x^{2} / 16} d x d s \leq C M=C
$$


for $r \leq 1$ (recall the assumption $M=1$ ).

3. $\left.i i^{\circ}\right)$ To estimate $I_{32}$ we introduce $v(x, s)=u(x, s)-m(s)$ and split the integral into

$$
I_{32}=\iint_{S_{r}} v \mathcal{B} \nabla v \nabla G+\iint_{S_{r}} m(s) \mathcal{B} \nabla u \nabla G=I_{321}+I_{322} .
$$

(Notice that $\nabla v=\nabla u$.)

3.ii. $\left.a^{\circ}\right) I_{321}$ is estimated as in [CK98, p.404]. Let

$$
\begin{aligned}
E_{3}(s) & =\int_{\mathbb{R}^{n}} v \mathcal{B} \nabla v \nabla G(x,-s)=-\int_{\mathbb{R}^{n}} v \mathcal{B} \nabla v \frac{x}{2|s|} G(x,-s) \\
& =\int_{|x| \geq|s|^{1 / 4}}+\int_{|x| \leq|s|^{1 / 4}}=: E_{31}(s)+E_{32}(s) .
\end{aligned}
$$

3.ii.a. $\alpha^{\circ}$ ) Applying Cauchy-Schwarz and then Poincaré inequalities, we obtain

$$
\begin{aligned}
\left|E_{31}(s)\right| & \leq C\left(\int_{\mathbb{R}^{n}} v^{2} G(x,-s)\right)^{1 / 2}\left(\int_{|x| \geq|s|^{1 / 4}}|\nabla v|^{2} \frac{|x|^{2}}{|s|^{2}} G(x,-s)\right)^{1 / 2} \\
& \leq C|s|^{1 / 2}\left(\int_{\mathbb{R}^{n}}|\nabla v|^{2} G(x,-s)\right)^{1 / 2}\left(\int_{|x| \geq|s|^{1 / 4}}|\nabla v|^{2} \frac{|x|^{2}}{|s|^{2}} G(x,-s)\right)^{1 / 2} \\
& \leq C_{n} e^{-c_{0} /|s|^{1 / 2}} \int_{\mathbb{R}^{n}}|\nabla v|^{2} e^{-x^{2} / 8} d x
\end{aligned}
$$

where, we have used that

$$
\begin{array}{ll}
G(x,-s) \leq C_{n}|s|^{-n / 2} e^{-x^{2} / 4} & \text { for any }(x, s) \in S_{1} \\
\frac{|x|^{2}}{|s|^{2}} G(x,-s) \leq C_{n} e^{-c_{0} /|s|^{1 / 2}} e^{-x^{2} / 8} & \text { for }|x| \geq|s|^{1 / 4},-1<s<0
\end{array}
$$

But then

$$
\int_{-r^{2}}^{0}\left|E_{31}(s)\right| d s \leq C e^{-c_{0} / r} \iint_{S_{r}}|\nabla u|^{2} e^{-x^{2} / 8} d x d s \leq C e^{-c_{0} / r},
$$

where we have used the Energy Inequality, as in Step 3.i.

3.ii.a. $\beta^{\circ}$ ) Further

$$
\begin{aligned}
\left|E_{32}(s)\right| & \leq \omega\left(|s|^{1 / 4}\right)\left(\int_{\mathbb{R}^{n}} v^{2} \frac{|x|^{2}}{4|s|^{2}} G(x,-s)\right)^{1 / 2}\left(\int_{\mathbb{R}^{n}}|\nabla v|^{2} G(x,-s)\right)^{1 / 2} \\
& \leq C_{n} \omega\left(|s|^{1 / 4}\right) \int_{\mathbb{R}^{n}}|\nabla v|^{2} G(x,-s),
\end{aligned}
$$

provided we use following claim.

Claim.

$$
\int_{\mathbb{R}^{n}} v^{2} \frac{|x|^{2}}{|s|^{2}} d \gamma^{s} \leq C_{n} \int_{\mathbb{R}^{n}}|\nabla v|^{2} d \gamma^{s}
$$


Proof. We have,

$$
\begin{aligned}
\int_{\mathbb{R}^{n}} v^{2} \frac{|x|^{2}}{|s|} G(x,-s)= & -2 \int_{\mathbb{R}^{n}} v^{2} x \nabla G(x,-s) \\
= & 2 n \int_{\mathbb{R}^{n}} v^{2} G(x,-s)+4 \int_{\mathbb{R}^{n}} v \nabla v x G(x,-s) \\
\leq & 2 n \int_{\mathbb{R}^{n}} v^{2} G(x,-s) \\
& +4\left(\int_{\mathbb{R}^{n}} v^{2} \frac{|x|^{2}}{|s|} G(x,-s)\right)^{1 / 2}\left(|s| \int_{\mathbb{R}^{n}}|\nabla v|^{2} G(x,-s)\right)^{1 / 2} .
\end{aligned}
$$

Then, we use the Poincaré inequality Lemma 2.3 to finish the proof.

Collecting the estimates for $E_{31}(s)$ and $E_{32}(s)$, integrating, and using that $e^{-C / r} \leq C r^{4}$, we obtain

$$
\left|I_{321}\right| \leq C r^{4}+C_{n} \omega\left(r^{1 / 2}\right) \iint_{S_{r}}|\nabla u|^{2} d \gamma
$$

3.ii. $\left.b^{\circ}\right)$ Further, write

$$
I_{322}=\int_{-r^{2}}^{0} m(s) E_{2}(s) d s
$$

and recall the estimates from $2 . \mathrm{i}^{\circ}-2 . \mathrm{ii}^{\circ}$ :

$$
m(s) \leq m\left(-r^{2}\right)+r^{2}+C_{n} \theta(r)\left(\iint_{S_{r}}|\nabla u|^{2} d \gamma\right)^{1 / 2}
$$

for $-r^{2}<s \leq 0$. The proof of the estimate above contains also the following inequality

$$
\int_{-r^{2}}^{0}\left|E_{2}(s)\right| d s \leq C_{n} \theta(r)\left(\iint_{S_{r}}|\nabla u|^{2} d \gamma\right)^{1 / 2} .
$$

Combining these estimates and using the Cauchy-Schwarz again, we obtain

$$
\begin{aligned}
\left|I_{322}\right| \leq & C_{n} \theta(r)^{2} \iint_{S_{r}}|\nabla u|^{2} d \gamma+C_{n} r^{2} \theta(r)\left(\iint_{S_{r}}|\nabla u|^{2} d \gamma\right)^{1 / 2} \\
& +C_{n} m\left(-r^{2}\right) \theta(r)\left(\iint_{S_{r}}|\nabla u|^{2} d \gamma\right)^{1 / 2} \\
\leq & C_{n} r^{4}+C_{n} m\left(-r^{2}\right)^{2} \theta(r)+C_{n}\left(\theta(r)^{2}+\theta(r)\right) \iint_{S_{r}}|\nabla u|^{2} d \gamma .
\end{aligned}
$$

Thus, we have

$$
\left|I_{322}\right| \leq C r^{4}+C_{n} \theta(r) m\left(-r^{2}\right)^{2}+C_{n} \theta(r) \iint_{S_{r}}|\nabla u|^{2} d \gamma .
$$

Collecting the estimates in $3 . \mathrm{i}^{-}-3 . \mathrm{ii}^{\circ}$ we also obtain

$$
\left|I_{3}\right| \leq C r^{4}+C_{n} \theta(r) m\left(-r^{2}\right)^{2}+C_{n} \theta(r) \iint_{S_{r}}|\nabla u|^{2} d \gamma .
$$


$4^{\circ}$ ) Finally, we estimate

$$
I_{4}=-\iint_{S_{r}} u b \nabla u G(x,-s) d x d s
$$

We use approach similar to the estimation of $I_{3}$. Let $v(x, s)=u(x, s)-m(s)$, as before, and split

$$
I_{4}=-\iint_{S_{r}} v b \nabla v G-\iint_{S_{r}} m(s) b \nabla v G=: I_{41}+I_{42} .
$$

$\left.4 . i^{\circ}\right)$ Using Cauchy-Schwarz and Poincaré inequalities, we obtain

$$
\begin{aligned}
\left|I_{41}\right| & \leq \mu \iint_{S_{r}}|v||\nabla v| d \gamma \\
& \leq \mu\left(\iint_{S_{r}}|v|^{2} d \gamma\right)^{1 / 2}\left(\iint_{S_{r}}|\nabla v|^{2} d \gamma\right)^{1 / 2} \\
& \leq C r \iint_{S_{r}}|\nabla v|^{2} d \gamma=C r \iint_{S_{r}}|\nabla u|^{2} d \gamma .
\end{aligned}
$$

4..$\left.i i^{\circ}\right)$ Further, we have

$$
\begin{aligned}
\left|I_{42}\right| & \leq \mu \int_{-r^{2}}^{0} m(s)\left(\int_{\mathbb{R}^{n}}|\nabla u|^{2} d \gamma^{s}\right)^{1 / 2} d s \\
& \leq \mu\left[\sup _{\left(-r^{2}, 0\right]} m(s)\right] r\left(\iint_{S_{r}}|\nabla u|^{2} d \gamma\right)^{1 / 2} .
\end{aligned}
$$

Recalling the estimate

$$
m(s) \leq m\left(-r^{2}\right)+r^{2}+C_{n} \theta(r)\left(\iint_{S_{r}}|\nabla u|^{2} d \gamma\right)^{1 / 2}
$$

for $-r^{2}<s \leq 0$, we therefore obtain

$$
\begin{aligned}
\left|I_{42}\right| & \leq \mu\left[m\left(-r^{2}\right) r+r^{3}\right]\left(\iint_{S_{r}}|\nabla u|^{2} d \gamma\right)^{1 / 2}+C r \theta(r) \iint_{S_{r}}|\nabla u|^{2} d \gamma \\
& \leq C m\left(-r^{2}\right)^{2} r+C r^{4}+\left[C r^{2}+r+\theta(r)^{2}\right] \iint_{S_{r}}|\nabla u|^{2} d \gamma \\
& \leq C m\left(-r^{2}\right)^{2} r+C r^{4}+\theta(r) \iint_{S_{r}}|\nabla u|^{2} d \gamma .
\end{aligned}
$$

Thus, collecting the estimates in $4 . \mathrm{i}^{\circ}-4 . \mathrm{ii}^{\circ}$, we conclude

$$
\left|I_{4}\right| \leq C r^{4}+\theta(r) m\left(-r^{2}\right)^{2}+\theta(r) \iint_{S_{r}}|\nabla u|^{2} d \gamma
$$

$5^{\circ}$ ) Combining all the estimates in $1^{\circ}-4^{\circ}$, we arrive at

$$
\begin{aligned}
\iint_{S_{r}}|\nabla u|^{2} d \gamma \leq & \frac{1+C_{n} \theta(r)}{2} \int_{\mathbb{R}^{n}} u\left(\cdot,-r^{2}\right)^{2} d \gamma^{-r^{2}}+C r^{4} \\
& +r^{2} m\left(-r^{2}\right)+C_{n} \theta(r) \iint_{S_{r}}|\nabla u|^{2} d \gamma
\end{aligned}
$$


Dividing by $1+C_{n} \theta(r)$, and assuming that $r<r_{\omega}$ is small enough, we obtain

$$
\left(1-C_{n} \theta(r)\right) \iint_{S_{r}}|\nabla u|^{2} d \gamma \leq \frac{1}{2} \int_{\mathbb{R}^{n}} u\left(\cdot,-r^{2}\right)^{2} d \gamma^{-r^{2}}+C r^{4}+C_{n} r^{2} m\left(-r^{2}\right) .
$$

Finally, applying the Hölder inequality

$$
m\left(-r^{2}\right) \leq\left(\int_{\mathbb{R}^{n}} u\left(\cdot,-r^{2}\right)^{2} d \gamma^{-r^{2}}\right)^{1 / 2},
$$

we complete the proof of Proposition 3.1.

We will also need the following simple corollary from Proposition 3.1.

Proposition 3.1'. If $u$ is as in Proposition 3.1, then for $0<r<r_{\omega}$ we also have

$$
\begin{aligned}
& \iint_{S_{r}}|\nabla u|^{2} d \gamma \leq C_{0} r^{4}+C_{0} \inf _{s \in\left[-4 r^{2},-r^{2}\right]} \int_{\mathbb{R}^{n}} u(\cdot, s)^{2} d \gamma^{s} \\
& \iint_{S_{r}}|\nabla u|^{2} d \gamma \leq C_{0} r^{4}+\frac{C_{0}}{r^{2}} \iint_{S_{2 r} \backslash S_{r}} u^{2} d \gamma .
\end{aligned}
$$

The proof of the next two propositions is based on the key Proposition 3.1 combined with the log-Sobolev inequality (see Lemma 2.2). It is not much different from the case when $\mathcal{L}=\Delta-\partial_{s}$, detailed proof of which can be found in [EP08]. Therefore, the proofs are omitted.

Proposition 3.2 (cf. [EP08, Propositions 1.2 and 2.2]). Let $u(x, s)$ be as in Proposition 3.1 and $\Omega:=\{u>0\}$. Suppose

$$
\iint_{\Omega \cap S_{r}}|\nabla u|^{2} d \gamma=\alpha r^{4}<\infty
$$

and

$$
\iint_{\Omega \cap S_{r / 4}}|\nabla u|^{2} d \gamma \geq \frac{\alpha r^{4}}{256}
$$

for some $0<r \leq r_{\omega}$. Then

$$
\left|\Omega \cap\left(S_{r / 2} \backslash S_{r / 4}\right)\right| \geq c_{0} r^{2}>0,
$$

provided $\alpha>\alpha_{0}$ for sufficiently large $\alpha_{0}$. (Here $|E|=\gamma(E)=\iint_{E} d \gamma$, for $E \subset$ $\mathbb{R}^{n} \times(-\infty, 0)$.)

Proposition 3.3 (cf. [EP08, Propositions 1.3 and 2.3]). Let $u(x, s)$ be as in Proposition 3.1 and $\Omega:=\{u>0\}$. Suppose

$$
\iint_{\Omega \cap S_{r}}|\nabla u|^{2} d \gamma=\alpha r^{4}<\infty
$$

for some $0<r \leq r_{\omega}$. Suppose also there exists $\eta>0$ such that

$$
\left|\Omega \cap\left(S_{r / 2} \backslash S_{r / 4}\right)\right|<(1-\eta)\left|S_{r / 2} \backslash S_{r / 4}\right| .
$$

Then there exists $\beta=\beta_{\eta}<1$ such that

$$
\iint_{\Omega \cap S_{r / 4}}|\nabla u|^{2} d \gamma \leq \beta \iint_{\Omega \cap S_{r}}|\nabla u|^{2} d \gamma,
$$

provided $\alpha>\alpha_{0}$ for sufficiently large $\alpha_{0}$. 
In the next proposition, we let

$$
\tilde{A}_{ \pm}(r)=e^{c_{0} g(r)} A_{ \pm}(r), \quad g(r)=\int_{0}^{r} \frac{\theta(\rho)}{\rho} d \rho
$$

for a universal constant $c_{0}>0$ to be determined, and

$$
\tilde{\Phi}(r)=r^{-4} \tilde{A}_{+}(r) \tilde{A}_{-}(r) .
$$

Notice that since we assume the double Dini condition on $\omega(r), \theta(r)$ satisfies the Dini condition and therefore $g(r)$ is finite and converges to 0 as $r \rightarrow 0+$.

Proposition 3.4 (cf. [EP08, Propositions 1.4 and 2.4]). Let $u_{ \pm}$be as in Theorem I with $M_{ \pm} \leq 1$ and $\tilde{A}_{ \pm}, \tilde{\Phi}$ as defined above. Then there exists a universal constant $C_{0}$ such that if $\tilde{A}_{ \pm}(\rho) \geq C_{0} r^{4}$ for all $\rho \in\left[\frac{1}{4} r, r\right], 0<r \leq r_{\omega}$, then

$$
\tilde{\Phi}^{\prime}(\rho) \geq-C_{0} r\left[\frac{1}{\sqrt{\tilde{A}_{+}(\rho)}}+\frac{1}{\sqrt{\tilde{A}_{-}(\rho)}}\right] \tilde{\Phi}(\rho)
$$

for all $\rho \in\left[\frac{1}{4} r, r\right]$.

Remark. We may replace $\tilde{A}_{ \pm}$by $A_{ \pm}$in this proposition, since the factor $e^{c_{0} g(r)}$ is bounded away from 0 and $\infty$. Yet, we must take the derivative of $\tilde{\Phi}$ to compensate for having $\theta(r)$ in Proposition 3.1.

Proof. We start with the same remark as in the proof of Lemma 2.4 in [CJK02]. The functions $A_{ \pm}$are continuous nondecreasing functions, hence $\Phi^{\prime}$ is the sum of a nonnegative singular measure and an absolutely continuous part and we need to obtain the bound on $\Phi^{\prime}$ at the points $r$ that are Lebesgue points for the integrands of $A_{ \pm}$. Thus, we assume that $r$ is such that

$$
B_{ \pm}(r)=\int_{\mathbb{R}^{n}}\left|\nabla u_{ \pm}\left(\cdot,-r^{2}\right)\right|^{2} d \gamma^{-r^{2}}<\infty
$$

and that $A_{ \pm}^{\prime}(r)=2 r B_{ \pm}(r)$.

We then have

$$
\begin{aligned}
\frac{\tilde{\Phi}^{\prime}(r)}{\tilde{\Phi}(r)} & =2 c_{0} g^{\prime}(r)-\frac{4}{r}+2 r \frac{B_{+}(r)}{A_{+}(r)}+2 r \frac{B_{-}(r)}{A_{-}(r)} \\
& =\frac{1}{r}\left(-4+2 c_{0} \theta(r)+2 r^{2} \frac{B_{+}(r)}{A_{+}(r)}+2 r^{2} \frac{B_{-}(r)}{A_{-}(r)}\right)
\end{aligned}
$$

Next, by Proposition 3.1, we have

$$
2\left(1-c_{n} \theta(r)\right) A_{+}(r) \leq C r^{4}+C r^{2}\left[\int_{\mathbb{R}^{n}} u_{+}\left(\cdot,-r^{2}\right)^{2} d \gamma^{-r^{2}}\right]^{1 / 2}+\int_{\mathbb{R}^{n}} u_{+}\left(\cdot,-r^{2}\right)^{2} d \gamma^{-r^{2}}
$$

for a universal constant $C$. Before we proceed, observe that $u_{+}\left(\cdot,-r^{2}\right)$ cannot vanish identically on $\mathbb{R}^{n}$ if the constant $C_{0}$ in the statement of Proposition 3.4 is sufficiently large. Indeed, otherwise we would have $A_{+}(r) \leq \mathrm{Cr}^{4}$ from Proposition 3.1 , which would be a contradiction. Similarly, $u_{-}\left(\cdot,-r^{2}\right)$ cannot vanish identically on $\mathbb{R}^{n}$. Then

$$
\Omega_{ \pm}^{-r^{2}}=\left\{u_{ \pm}\left(\cdot,-r^{2}\right)>0\right\}
$$


are nonempty. If we now denote $\lambda_{ \pm}=r^{2} \lambda^{-r^{2}}\left(\Omega_{ \pm}^{-r^{2}}\right)$, the normalized eigenvalues of $\Omega_{ \pm}^{-r^{2}}$ as defined in Lemma 2.4, we will have

$$
\lambda_{+}+\lambda_{-} \geq 1
$$

Next, by the definition of $\lambda_{ \pm}$we have

$$
\lambda_{ \pm} \int_{\Omega_{ \pm}^{-r^{2}}} f^{2} d \gamma^{-r^{2}} \leq r^{2} \int_{\Omega_{ \pm}^{-r^{2}}}|\nabla f|^{2} d \gamma^{-r^{2}}
$$

Combining this with Proposition 3.1, we obtain

$$
2\left(1-c_{n} \theta(r)\right) A_{ \pm}(r) \leq C r^{4}+C r^{3} \sqrt{B_{ \pm}(r) / \lambda_{ \pm}}+r^{2} B_{ \pm}(r) / \lambda_{ \pm} .
$$

To complete the proof, we consider the following possibilities:

$\left.1^{\circ}\right) r^{2} B_{+}(r) \geq 2 A_{+}(r)\left(\right.$ or $\left.r^{2} B_{-}(r) \geq 2 A_{-}(r)\right)$. Then

$$
\frac{\tilde{\Phi}^{\prime}(r)}{\tilde{\Phi}(r)}=\frac{1}{r}\left(-4+2 c_{0} \theta(r)+2 r^{2} \frac{B^{+}}{A^{+}}+2 r^{2} \frac{B^{-}}{A^{-}}\right) \geq 0 .
$$

$\left.2^{\circ}\right) r^{2} B_{+}(r) \leq 2 A_{+}(r)$ and $\lambda_{+} \geq 1$ (or $r^{2} B_{-}(r) \leq 2 A_{-}(r)$ and $\left.\lambda_{-} \geq 1\right)$. Then by (3.9), if $A_{+}(r) \geq C_{0} r^{4}$ is sufficiently large, we have

$$
2\left(1-c_{n} \theta(r)\right) A_{+}(r) \leq C r^{2} \sqrt{A_{+}(r)}+r^{2} B_{+}(r) .
$$

It follows then

$$
\begin{aligned}
\frac{\tilde{\Phi}^{\prime}(r)}{\tilde{\Phi}(r)} & \geq \frac{1}{r}\left(-4+2 c_{0} \theta(r)+2 r^{2} \frac{B_{+}(r)}{A_{+}(r)}\right) \\
& \geq-\frac{2 C r}{\sqrt{A_{+}(r)}}+\frac{1}{r}\left(-4+2 c_{0} \theta(r)+4-4 c_{n} \theta(r)\right) \geq-\frac{2 C r}{\sqrt{A_{+}(r)}},
\end{aligned}
$$

if we choose $c_{0} \geq 2 c_{n}$.

$\left.3^{\circ}\right) r^{2} B_{ \pm}(r) \leq 2 A_{ \pm}(r)$ and $\lambda_{ \pm} \leq 1$. Then by (3.9), if $A_{ \pm}(r) \geq C_{0} r^{4}$ are sufficiently large, we have

$$
2 \lambda_{ \pm}\left(1-c_{n} \theta(r)\right) A_{ \pm}(r) \leq C r^{2} \sqrt{A^{ \pm}(r)}+r^{2} B_{ \pm}(r) .
$$

If we use now that $\lambda_{+}+\lambda_{-} \geq 1$ and choose $c_{0} \geq 2 c_{n}$, then

$$
\begin{aligned}
\frac{\tilde{\Phi}^{\prime}(r)}{\tilde{\Phi}(r)} & =\frac{1}{r}\left(-4+2 c_{0} \theta(r)+2 r^{2} \frac{B_{+}(r)}{A_{+}(r)}+2 r^{2} \frac{B_{-}(r)}{A_{-}(r)}\right) \\
& \geq \frac{1}{r}\left(-4+2 c_{0} \theta(r)+4\left(\lambda_{+}+\lambda_{-}\right)\left(1-c_{n} \theta(r)\right)-C r^{2}\left[\frac{1}{\sqrt{A_{+}(r)}}+\frac{1}{\sqrt{A_{-}(r)}}\right]\right) \\
& \geq-C r\left[\frac{1}{\sqrt{A_{+}(r)}}+\frac{1}{\sqrt{A_{-}(r)}}\right] .
\end{aligned}
$$

In the same way we prove the estimate for $\tilde{\Phi}^{\prime}(\rho)$ for any $\rho \in\left[\frac{1}{4} r, r\right]$ and the proof is complete. 
As we mentioned before, the propositions above constitute the technical core of the proof of Theorem I. The rest of the proof is purely arithmetic in nature and is exactly the same as in [CJK02]. We consider a geometric sequence of radii $r=4^{-k}$, $k=0,1,2, \ldots$, and define

$$
\tilde{A}_{k}^{ \pm}=\tilde{A}_{ \pm}\left(4^{-k}\right), \quad \tilde{b}_{k}^{ \pm}=4^{4 k} \tilde{A}_{k}^{ \pm},
$$

where $\tilde{A}_{ \pm}(r)=e^{c_{0} g(r)} A_{ \pm}(r)$ are as in Propositions 3.4 , so that we have

$$
\tilde{\Phi}\left(4^{-k}\right)=4^{4 k} \tilde{A}_{k}^{+} \tilde{A}_{k}^{-}=A_{k}^{+} \tilde{b}_{k}^{-}=A_{k}^{-} \tilde{b}_{k}^{+}
$$

One may also treat $\tilde{b}_{k}^{ \pm}$as the correctly rescaled versions of $\tilde{A}_{k}^{ \pm}$because of the the following property: if we consider the parabolic scaling $u_{r}(x, s):=r^{-2} u\left(r x, r^{2} s\right)$, then

$$
A\left(1, u_{r}\right)=\iint_{S_{1}}\left|\nabla u_{r}\right|^{2} d \gamma=r^{-4} \iint_{S_{r}}|\nabla u|^{2} d \gamma=r^{-4} A(r, u) .
$$

The proofs of the next two propositions are the same as those of [CJK02, Lemmas 2.8-2.9], based on Propositions 3.2-3.4 instead of [CJK02, Lemmas 2.1-2.3], and therefore are omitted.

Proposition 3.5 (cf. [EP08, Propositions 1.5 and 2.5]). Let $u_{ \pm}$be as in Theorem I with $M_{ \pm} \leq 1$. There exists a universal constant $C_{0}$ such that if $\tilde{b}_{k}^{ \pm} \geq C_{0}$ for $k \geq k_{\omega}$ then

$$
4^{4} \tilde{A}_{k+1}^{+} \tilde{A}_{k+1}^{-} \leq \tilde{A}_{k}^{+} \tilde{A}_{k}^{-}\left(1+\delta_{k}\right) \quad \text { with } \quad \delta_{k}=\frac{C_{0}}{\sqrt{\tilde{b}_{k}^{+}}}+\frac{C_{0}}{\sqrt{\tilde{b}_{k}^{-}}} .
$$

Proposition 3.6 (cf. [EP08, Propositions 1.6 and 2.6]). Let $u_{ \pm}$be as in Theorem I with $M_{ \pm} \leq 1$. There exists a universal constant $\varepsilon_{0}>0$, such that if $\tilde{b}_{k}^{ \pm} \geq C_{0}$ and $4^{4} \tilde{A}_{k+1}^{+} \geq \tilde{A}_{k}^{+}$for $k \geq k_{\omega}$, then $\tilde{A}_{k+1}^{-} \leq\left(1-\varepsilon_{0}\right) \tilde{A}_{k}^{-}$.

Proof of Theorem I. Recalling the initial reduction steps in Subsection 3.1, we need to show that

$$
\Phi\left(r, u_{+}, u_{-}\right) \leq C_{\omega}, \quad \text { for } r \leq r_{\omega}
$$

Now, Propositions 3.5-3.6 provide the same iteration scheme (starting from $k=k_{\omega}$ ) as in [CJK02] which implies that

$$
\Phi\left(r, u_{+}, u_{-}\right) \leq C_{\omega}\left(1+A_{+}\left(4^{-k_{\omega}}\right)+A_{-}\left(4^{-k_{\omega}}\right)\right)^{2}, \quad r \leq 4^{-k_{\omega}}
$$

Finally, note that by Proposition $3.1^{\prime} A_{ \pm}\left(4^{-k_{\omega}}\right) \leq C$, which completes the proof of the theorem.

\section{Localized Parabolic Formula}

In this section we prove Theorem II. The proof is essentially the same as in the global case, i.e. Theorem I, but has to take into account additional error terms coming from the cutoff function $\psi$. Those new error terms, as we will see, are actually exponentially small, since they are basically the integrals over the tails of Gaussian function $G(x,|s|)$ for $|x| \geq 1 / 2$.

Proposition 4.1 (cf. Proposition 3.1). Let $u \in C\left(Q_{1}^{-}\right) \cap V^{2}\left(Q_{1}^{-}\right)$satisfy

$$
u \geq 0, \quad \mathcal{L}_{\mathcal{A}, b, 0} u \geq-1 \quad \text { in } Q_{1}^{-} .
$$


Suppose also

$$
\iint_{Q_{1}^{-}} u(x, s)^{2} d x d s \leq 1 .
$$

Let $\psi \in C^{\infty}\left(B_{1}\right)$ be such that $0<\psi \leq 1,\left.\psi\right|_{B_{1} / 2}=1$ and define

$$
w(x, s)=u(x, s) \psi(x), \quad(x, s) \in Q_{1}^{-} .
$$

Then

$$
\begin{aligned}
\left(1-c_{n} \theta(r)\right) \iint_{S_{r}}|\nabla w|^{2} d \gamma & \leq C_{\psi} r^{4}+C_{n} r^{2}\left(\int_{\mathbb{R}^{n}} w\left(\cdot,-r^{2}\right)^{2} d \gamma^{-r^{2}}\right)^{1 / 2} \\
& +\frac{1}{2} \int_{\mathbb{R}^{n}} w\left(\cdot,-r^{2}\right)^{2} d \gamma^{-r^{2}}
\end{aligned}
$$

for any $0<r \leq r_{\omega}$, where

$$
\theta(r)=C r+\omega\left(r^{1 / 2}\right)+\left(\int_{0}^{r^{2}} \frac{\omega\left(\rho^{1 / 4}\right)^{2}}{\rho} d \rho\right)^{1 / 2} .
$$

Proof. Let $w(x, s)=u(x, s) \psi(x)$. Then for $\mathcal{L}=\mathcal{L}_{\mathcal{A}, b, 0}$, we have

$$
\begin{aligned}
\left(\Delta-\partial_{s}\right) w= & \mathcal{L} w+\operatorname{div}(\mathcal{B} \nabla w)-b \nabla w \\
= & \psi \mathcal{L} u+u \mathcal{L} \psi+2 \nabla \psi \mathcal{A} \nabla u+\operatorname{div}(\mathcal{B} \nabla w)-b \nabla w \\
\geq & -1+\operatorname{div}(\mathcal{B} \nabla w)-b \nabla w+u \mathcal{L} \psi+2 \nabla \psi \mathcal{A} \nabla u \\
\left(\Delta-\partial_{s}\right)\left(w^{2} / 2\right) \geq & w[-1+\operatorname{div}(\mathcal{B} \nabla w)-b \nabla w+u \mathcal{L} \psi \\
& +2 \nabla \psi \mathcal{A} \nabla u]+|\nabla w|^{2} \\
\geq & -w+w \operatorname{div}(\mathcal{B} \nabla w)-w b \nabla w+w u \mathcal{L} \psi \\
& +2 w \nabla \psi \mathcal{A} \nabla u+|\nabla w|^{2},
\end{aligned}
$$

in the sense of distributions. Then (4.2) implies

$$
\begin{aligned}
\iint_{S_{r}}|\nabla w|^{2} d \gamma \leq & \frac{1}{2} \iint_{S_{r}}\left(\Delta-\partial_{s}\right)\left(w^{2}\right) d \gamma+\iint_{S_{r}} w d \gamma-\iint_{S_{r}} w \operatorname{div}(\mathcal{B} \nabla w) d \gamma \\
& +\iint_{S_{r}} w b \nabla w d \gamma+\iint_{S_{r}}(w u \mathcal{L} \psi+2 w \nabla \psi \mathcal{A} \nabla u) d \gamma \\
= & : \frac{1}{2} I_{1}+I_{2}+I_{3}+I_{4}+I_{5} .
\end{aligned}
$$

The terms $I_{1}, \ldots, I_{4}$ are estimated very similarly to the global case, however, there are some minor differences and we prefer to provide more details. The term $I_{5}$ is new, but we are going to see that its contribution is exponentially small.

$\left.1^{\circ}\right)$ Exactly as in the global case, we have

$$
I_{1} \leq \int_{\mathbb{R}^{n}} w\left(\cdot,-r^{2}\right)^{2} d \gamma^{-r^{2}} .
$$

$2^{\circ}$ ) To estimate $I_{2}$, consider the weighted averages of $w(\cdot, s)$

$$
m(s)=\int_{\mathbb{R}^{n}} w(\cdot, s) d \gamma^{s}=\int_{\mathbb{R}^{n}} w(x, s) G(x,-s) d x .
$$


Then, for $-r^{2} \leq s_{2} \leq s_{1} \leq 0$, using (4.1) and that $\left(\Delta+\partial_{s}\right) G(x,-s)=0$, we have

$$
\begin{aligned}
m\left(s_{1}\right)-m\left(s_{2}\right) & =-\int_{s_{2}}^{s_{1}} \int_{\mathbb{R}^{n}}\left(\Delta-\partial_{s}\right) w(x) G(x,-s) \\
& \leq \int_{s_{2}}^{s_{1}} \int_{\mathbb{R}^{n}}(1-\operatorname{div}(\mathcal{B} \nabla w)+b \nabla w-u \mathcal{L} \psi-2 \nabla \psi \mathcal{A} \nabla u) G \\
& =\left(s_{1}-s_{2}\right)+\int_{s_{2}}^{s_{1}} \int_{\mathbb{R}^{n}} \mathcal{B} \nabla w \nabla G+b \nabla w G-(u \mathcal{L} \psi+2 \nabla \psi \mathcal{A} \nabla u) G \\
& =\left(s_{1}-s_{2}\right)+\int_{s_{2}}^{s_{1}}\left(E_{2}(s)+H_{2}(s)+K_{2}(s)\right) d s
\end{aligned}
$$

where

$$
\begin{aligned}
E_{2}(s) & :=\int_{\mathbb{R}^{n}} \mathcal{B} \nabla w \nabla G(x,-s), \\
H_{2}(s) & :=\int_{\mathbb{R}^{n}} b \nabla w G(x,-s), \\
K_{2}(s) & :=-\int_{\mathbb{R}^{n}}(u \mathcal{L} \psi+2 \nabla \psi \mathcal{A} \nabla u) G(x,-s) .
\end{aligned}
$$

2. $i^{\circ}$ ) Arguing exactly as in the global case, we obtain

$$
\begin{aligned}
& \left|E_{2}(s)\right| \leq C_{n}\left(C+\frac{\omega\left(|s|^{1 / 4}\right)}{|s|^{1 / 2}}\right)\left(\int_{\mathbb{R}^{n}}|\nabla w|^{2} d \gamma^{s}\right)^{1 / 2}, \\
& \left|H_{2}(s)\right| \leq C\left(\int_{\mathbb{R}^{n}}|\nabla w|^{2} d \gamma^{s}\right)^{1 / 2} .
\end{aligned}
$$

Further, to estimate $K_{2}(s)$, integrate by parts the first term

$$
\begin{aligned}
K_{2}(s) & =-\int_{\mathbb{R}^{n}}(u \operatorname{div}(\mathcal{A} \nabla \psi)+u b \nabla \psi+2 \nabla \psi \mathcal{A} \nabla u) G \\
& =\int_{\mathbb{R}^{n}} \nabla u \mathcal{A} \nabla \psi G+u \mathcal{A} \nabla \psi \nabla G-u b \nabla \psi G-2 \nabla \psi \mathcal{A} \nabla u G
\end{aligned}
$$

and note that the cutoff function $\psi$ appears in the latter integral only in the form of $\nabla \psi$, which vanishes on $B_{1 / 2}$. Therefore, using the Energy Inequality and the fact that $G(x,-s)$ and $|\nabla G(x,-s)|$ are bounded above by $e^{-c_{0} /|s|}$ for $|x| \geq 1 / 2$, we easily obtain that

$$
\left|K_{2}(s)\right| \leq C_{\psi} e^{-c_{0} /|s|}
$$

2. $\left.i i^{\circ}\right)$ We continue the estimation of $m(s)$. Using the estimates on $\left|E_{2}(s)\right|,\left|H_{2}(s)\right|$ and $\left|K_{2}(s)\right|$ in $2 . \mathrm{i}^{\circ}$ and applying the Cauchy-Schwarz inequality, for $-r^{2} \leq s \leq 0$ we obtain

$$
\begin{aligned}
m(s) & \leq m\left(-r^{2}\right)+C_{\psi} r^{2}+C_{n} \int_{-r^{2}}^{0}\left(C+\frac{\omega\left(|s|^{1 / 4}\right)}{|s|^{1 / 2}}\right)\left(\int_{\mathbb{R}^{n}}|\nabla w|^{2} d \gamma^{s}\right)^{1 / 2} d s \\
& \leq m\left(-r^{2}\right)+C_{\psi} r^{2}+C_{n} \theta(r)\left(\iint_{S_{r}}|\nabla w|^{2} d \gamma\right)^{1 / 2} .
\end{aligned}
$$


Integrating one more time gives

$$
\begin{aligned}
I_{2}=\iint_{S_{r}} w d \gamma & =\int_{-r^{2}}^{0} m(s) d s \leq r^{2} m\left(-r^{2}\right)+C_{\psi} r^{4}+C_{n} r^{2} \theta(r)\left(\iint_{S_{r}}|\nabla w|^{2} d \gamma\right)^{1 / 2} \\
& \leq r^{2} m\left(-r^{2}\right)+C_{\psi} r^{4}+C_{n} r^{4}+\theta(r)^{2} \iint_{S_{r}}|\nabla w|^{2} d \gamma \\
& \leq r^{2} m\left(-r^{2}\right)+C_{\psi} r^{4}+\theta(r) \iint_{S_{r}}|\nabla w|^{2} d \gamma .
\end{aligned}
$$

That is,

$$
I_{2} \leq r^{2} m\left(-r^{2}\right)+C_{\psi} r^{4}+\theta(r) \iint_{S_{r}}|\nabla w|^{2} d \gamma
$$

$3^{\circ}$ ) We now estimate

$$
\begin{aligned}
I_{3} & =-\iint_{S_{r}} w \operatorname{div}(\mathcal{B} \nabla w) d \gamma=\iint_{S_{r}} \nabla w \mathcal{B} \nabla w G+\iint_{S_{r}} w \mathcal{B} \nabla w \nabla G \\
& =: I_{31}+I_{32} .
\end{aligned}
$$

$\left.3 . i^{\circ}\right)$ Repeating the arguments as before, we obtain

$$
\begin{aligned}
\left|I_{31}\right| & \leq \iint_{|x| \leq|s|^{1 / 4}} \omega\left(|s|^{1 / 4}\right)|\nabla w|^{2} G(x,-s)+C \iint_{|x| \geq|s|^{1 / 4}}|\nabla w|^{2} G \\
& \leq \omega\left(r^{1 / 2}\right) \iint_{S_{r}}|\nabla w|^{2} d \gamma+C_{\psi} e^{-c_{0} / r},
\end{aligned}
$$

where in the estimation of the second integral over we have used that

$$
G(x,-s) \leq e^{-c_{0} /|s|^{1 / 2}}, \quad \text { for }|x| \geq|s|^{1 / 4}
$$

and that

$$
\iint_{S_{r}}|\nabla w|^{2} d x d s \leq C_{\psi} \iint_{Q_{1}} u^{2} d x d s \leq C_{\psi}
$$

for $r \leq 1 / 2$ (recall the assumption $M=1$ ) by the Energy Inequality (Lemma 2.1). 3. $\left.i i^{\circ}\right)$ To estimate $I_{32}$ we introduce $v(x, s)=w(x, s)-m(s)$ and split the integral into

$$
I_{32}=\iint_{S_{r}} v \mathcal{B} \nabla v \nabla G+\iint_{S_{r}} m(s) \mathcal{B} \nabla w \nabla G=I_{321}+I_{322} .
$$

(Notice that $\nabla v=\nabla w$.)

3.ii. $a^{\circ}$ ) Repeating the arguments in the global case, we estimate

$$
\left|I_{321}\right| \leq C_{\psi} r^{4}+C_{n} \omega\left(r^{1 / 2}\right) \iint_{S_{r}}|\nabla w|^{2} d \gamma
$$

3.ii. $b^{\circ}$ ) Further, to estimate $I_{322}$, we write it as

$$
I_{322}=\int_{-r^{2}}^{0} m(s) E_{2}(s) d s
$$

Arguing as in the global case, we obtain from $2 . \mathrm{i}^{\circ}$ that

$$
\int_{-r^{2}}^{0}\left|E_{2}(s)\right| d s \leq C_{n} \theta(r)\left(\iint_{S_{r}}|\nabla w|^{2} d \gamma\right)^{1 / 2},
$$


which combined with the uniform bound from $2 . \mathrm{ii}^{\circ}$

$$
m(s) \leq m\left(-r^{2}\right)+C_{\psi} r^{2}+C_{n} \theta(r)\left(\iint_{S_{r}}|\nabla w|^{2} d \gamma\right)^{1 / 2},
$$

for $-r^{2}<s \leq 0$, gives

$$
\left|I_{322}\right| \leq C_{\psi} r^{4}+C_{n} \theta(r) m\left(-r^{2}\right)^{2}+C_{n} \theta(r) \iint_{S_{r}}|\nabla w|^{2} d \gamma
$$

Now collecting the estimates in $3 . \mathrm{i}^{-}-3 . \mathrm{ii}^{\circ}$ we also obtain

$$
\left|I_{3}\right| \leq C_{\psi} r^{4}+C_{n} \theta(r) m\left(-r^{2}\right)^{2}+C_{n} \theta(r) \iint_{S_{r}}|\nabla w|^{2} d \gamma
$$

$\left.4^{\circ}\right)$ Next, we estimate

$$
I_{4}=-\iint_{S_{r}} w b \nabla w G(x,-s) d x d s .
$$

As before, we denote $v(x, s)=w(x, s)-m(s)$ and split the integral

$$
I_{4}=-\iint_{S_{r}} v b \nabla v G-\iint_{S_{r}} m(s) b \nabla v G=: I_{41}+I_{42} .
$$

Same arguments as in the global case prove that

$$
\begin{aligned}
& \left|I_{41}\right| \leq C r \iint_{S_{r}}|\nabla w|^{2} d \gamma \\
& \left|I_{42}\right| \leq C m\left(-r^{2}\right)^{2} r+C_{\psi} r^{4}+\theta(r) \iint_{S_{r}}|\nabla w|^{2} d \gamma
\end{aligned}
$$

which implies that

$$
\left|I_{4}\right| \leq C_{\psi} r^{4}+\theta(r) m\left(-r^{2}\right)^{2}+\theta(r) \iint_{S_{r}}|\nabla w|^{2} d \gamma
$$

$5^{\circ}$ ) Finally, we estimate

$$
I_{5}=\iint_{S_{r}}(w u \mathcal{L} \psi+2 w \nabla \psi \mathcal{A} \nabla u) d \gamma .
$$

Integrating by parts in space, we obtain

$$
\begin{aligned}
I_{5} & =\iint_{S_{r}} w u \operatorname{div}(\mathcal{A} \nabla \psi) G+w u b \nabla \psi G+2 w \nabla \psi \mathcal{A} \nabla u G \\
& =\iint_{S_{r}}-\nabla w u \mathcal{A} \nabla \psi G-w \nabla u \mathcal{A} \nabla \psi G-w u \mathcal{A} \nabla \psi \nabla G+w u b \nabla \psi G+2 w \nabla \psi \mathcal{A} \nabla u G .
\end{aligned}
$$

Every term in the last integrand contains $\nabla \psi$, which vanishes on $B_{1 / 2}$. Then using the Energy Inequality and the estimate on $G$ and $|\nabla G|$ for $|x| \geq 1 / 2$, we easily obtain that

$$
\left|I_{5}\right| \leq C_{\psi} e^{-c_{0} / r^{2}}
$$

$6^{\circ}$ ) Combining all the estimates in $1^{\circ}-5^{\circ}$, and using that

$$
m\left(-r^{2}\right)^{2} \leq \int_{\mathbb{R}^{n}} w\left(\cdot,-r^{2}\right)^{2} d \gamma^{-r^{2}}
$$


we arrive at

$$
\begin{aligned}
\iint_{S_{r}}|\nabla w|^{2} d \gamma \leq & \frac{1+C_{n} \theta(r)}{2} \int_{\mathbb{R}^{n}} w\left(\cdot,-r^{2}\right)^{2} d \gamma^{-r^{2}}+C_{\psi} r^{4} \\
& +r^{2} m\left(-r^{2}\right)+C_{n} \theta(r) \iint_{S_{r}}|\nabla w|^{2} d \gamma
\end{aligned}
$$

Dividing by $1+C_{n} \theta(r)$, and assuming that $r<r_{\omega}$ is small enough, we obtain

$$
\left(1-C_{n} \theta(r)\right) \iint_{S_{r}}|\nabla w|^{2} d \gamma \leq \frac{1}{2} \int_{\mathbb{R}^{n}} w\left(\cdot,-r^{2}\right)^{2} d \gamma^{-r^{2}}+C_{\psi} r^{4}+C_{n} r^{2} m\left(-r^{2}\right),
$$

as claimed. This completes the proof of Proposition 4.1.

Proof of Theorem II. Using Proposition 4.1 one may prove the analogues of Propositions 3.2-3.6, with obvious changes, possibly adding the dependence of constants on $\psi$. But then we argue as in the proof of Theorem I to complete the proof.

\section{Elliptic Formula}

In this section we prove Theorem III. Even though it possible to give a direct proof (by working on spheres as in [CJK02] instead of Gaussian spaces), we prefer to obtain the elliptic almost monotonicity formula from the localized parabolic one (Theorem II).

Proof of Theorem III. Let $u_{ \pm}(x)$ satisfy the assumptions of Theorem III. Adding a "dummy" variable $s$ by setting

$$
\tilde{u}_{ \pm}(x, s)=u_{ \pm}(x), \quad(x, s) \in Q_{1}^{-},
$$

we see that $\tilde{u}_{ \pm}$satisfy also the assumptions of Theorem II with the operator

$$
\mathcal{L} u=\left(\ell-\partial_{s}\right) u=\operatorname{div}(\mathcal{A}(x) \nabla u)+b(x) \nabla u+c(x) u-\partial_{s} u .
$$

Moreover, we claim that

$$
a\left(r, u_{ \pm}\right) \leq C_{n} A_{ \pm}\left(r, \psi \tilde{u}_{ \pm}\right), \quad r<1 / 2
$$

or in the expanded form

$$
\int_{B_{r}} \frac{|\nabla u(x)|}{|x|^{n-2}} d x \leq C_{n} \iint_{S_{r}}|\nabla(\psi(x) u(x))|^{2} G(x,-s) d x d s .
$$

Since $\psi=1$ on $B_{1 / 2}$, it is enough to show that

$$
\frac{c_{n}}{|x|^{n-2}} \leq \int_{0}^{r^{2}} G(x, t) d t, \quad \text { for } x \in B_{r} .
$$

Making a substitution $t=|x|^{2} \tau$, we obtain

$$
\begin{aligned}
\int_{0}^{r^{2}} G(x, t) d t & =C_{n} \int_{0}^{r^{2}} t^{-n / 2} e^{-|x|^{2} / 4 t} d t \\
& =\frac{C_{n}}{|x|^{n-2}} \int_{0}^{r^{2} /|x|^{2}} \tau^{-n / 2} e^{-1 / 4 \tau} d \tau \geq \frac{C_{n}}{|x|^{n-2}} \int_{0}^{1} \tau^{-n / 2} e^{-1 / 4 \tau} d \tau \\
& =\frac{c_{n}}{|x|^{n-2}} .
\end{aligned}
$$

Hence (5.1) follows. Consequently, we obtain that

$$
\varphi\left(r, u_{+}, u_{-}\right) \leq C_{n} \Phi\left(r, \psi \tilde{u}_{+}, \psi \tilde{u}_{-}\right) .
$$


Fixing a cutoff function $\psi$ and applying Theorem II, we obtain

$$
\begin{aligned}
\varphi\left(r, u_{+}, u_{-}\right) & \leq C_{\omega}\left(1+\left\|\tilde{u}_{+}\right\|_{L^{2}\left(Q_{1}^{-}\right)}^{2}+\left\|\tilde{u}_{-}\right\|_{L^{2}\left(Q_{1}^{-}\right)}^{2}\right)^{2} \\
& =C_{\omega}\left(1+\left\|u_{+}\right\|_{L^{2}\left(B_{1}\right)}^{2}+\left\|u_{-}\right\|_{L^{2}\left(B_{1}\right)}^{2}\right)^{2}
\end{aligned}
$$

for $r<r_{\omega}$. The theorem is proved.

\section{A Variant of the Formula}

Under assumptions on the growth of functions $u_{ \pm}$near the origin, following [CJK02, Theorem 3.8], it is possible to prove versions of Theorems I-III, where $\Phi(r)$ (and $\varphi(r))$ retain more monotonicity properties, e.g. that the limit $\Phi(0+)$ exists.

Here we state only the result in the localized parabolic case. We also assume that we are in the normalized case (3.1)-(3.3), as well as under the assumption $c=0$, which does not limit the generality.

Theorem IV (Almost Monotonicity Formula with Growth Assumption). Let $u_{ \pm}$, $\psi, w_{ \pm}, A_{ \pm}$and $\Phi$ be as in Theorem II. Assume additionally that

$$
u_{ \pm}(x, s) \leq \sigma\left(\left(|x|^{2}+|s|\right)^{1 / 2}\right) \quad \text { for }(x, s) \in Q_{1}^{-}
$$

for a Dini modulus of continuity $\sigma(r)$ (so that $\left.\int_{0}^{1} \frac{\sigma(\rho)}{\rho} d \rho<\infty\right)$. Then

$$
\Phi(r) \leq[1+\alpha(\rho)] \Phi(\rho)+C_{M, \psi, \sigma, \omega} \alpha(\rho), \quad 0<r \leq \rho \leq r_{\omega},
$$

where

$$
\begin{aligned}
& \alpha(r)=C_{0}\left[r+\sigma\left(r^{1 / 2}\right)+\int_{0}^{r} \frac{\sigma\left(\rho^{1 / 2}\right)}{\rho} d \rho+\int_{0}^{r} \frac{\theta(\rho)}{\rho} d \rho\right] \\
& \theta(r)=C_{0} r+\omega\left(r^{1 / 2}\right)+\left(\int_{0}^{r^{2}} \frac{\omega\left(\rho^{1 / 4}\right)^{2}}{\rho} d \rho\right)^{1 / 2},
\end{aligned}
$$

and $M=\left\|u_{+}\right\|_{L^{2}\left(Q_{1}^{-}\right)}+\left\|u_{-}\right\|_{L^{2}\left(Q_{1}^{-}\right)}$.

Remark. It is easy to see that the inequality above implies the existence of the limit $\Phi(0+)=\lim _{r \rightarrow 0+} \Phi(r)$.

Proof. As before, without loss of generality we may assume $M \leq 1$, otherwise we could consider $u_{ \pm} /(1+M)$. To simplify notations in this proof, we are going to deviate from our convention for constants and denote by $C$ a generic constant depending $\psi, \sigma, \omega$ in addition to $n$ and the structural constants in (1.2)-(1.4), which we would normally denote $C_{\psi, \sigma, \omega}$.

Notice that if $\Phi(r)=0$, then the estimate in the theorem is trivially satisfied. Therefore we will assume that $\Phi(r)>0$. Further, as in the proof of Proposition 3.4, we may assume $r$ to be a Lebesgue point for $B_{ \pm}$, which yields that

$$
A_{ \pm}^{\prime}(r)=2 r B_{ \pm}(r), \quad B_{ \pm}(r)=\int_{\mathbb{R}^{n}}\left|\nabla w_{ \pm}\left(\cdot,-r^{2}\right)\right|^{2} d \gamma^{-r^{2}}<\infty .
$$

Next, as in Proposition 3.4, we introduce

$$
\begin{gathered}
\tilde{A}_{ \pm}(r)=e^{c_{0} g(r)} A_{ \pm}(r), \quad g(r)=\int_{0}^{r} \frac{\theta(\rho)}{\rho} d \rho \\
\tilde{\Phi}(r)=r^{-4} \tilde{A}_{+}(r) \tilde{A}_{-}(r)=e^{2 c_{0} g(r)} \Phi(r) .
\end{gathered}
$$


Then we have

$$
\begin{aligned}
\frac{\tilde{\Phi}^{\prime}(r)}{\tilde{\Phi}(r)} & =2 c_{0} g^{\prime}(r)-\frac{4}{r}+2 r \frac{B_{+}(r)}{A_{+}(r)}+2 r \frac{B_{-}(r)}{A_{-}(r)} \\
& =\frac{1}{r}\left(-4+2 c_{0} \theta(r)+2 r^{2} \frac{B_{+}(r)}{A_{+}(r)}+2 r^{2} \frac{B_{-}(r)}{A_{-}(r)}\right) .
\end{aligned}
$$

To proceed, we are going to assume that

$$
\sigma\left(r^{1 / 2}\right) \geq r .
$$

This does not limit the generality, since we can replace $\sigma(\rho)$ with $\sigma(\rho)+\rho^{2}$ without affecting the form of $\alpha(r)$ in the statement of the theorem.

$1^{\circ}$ ) We now claim that the additional growth assumption on $u$ implies that

$$
A_{ \pm}(r) \leq C \sigma\left(r^{1 / 2}\right)^{2}, \quad r<r_{\omega}
$$

Indeed, let $w$ be either $w_{+}$or $w_{-}$. Then by Proposition 4.1, we have

$$
\begin{aligned}
\iint_{S_{r}}|\nabla w|^{2} d \gamma & \leq C r^{4}+C \int_{\mathbb{R}^{n}} w^{2}\left(x,-r^{2}\right) G\left(x, r^{2}\right) d x \\
& =C r^{4}+C \int_{|x|<r^{1 / 2}} w^{2} G\left(x,-r^{2}\right) d x+C \int_{|x|>r^{1 / 2}} w^{2} G\left(x,-r^{2}\right) d x \\
& \leq C r^{4}+C \sigma\left(r^{1 / 2}\right)^{2}+C e^{-c_{n} / r} \leq C \sigma\left(r^{1 / 2}\right)^{2} .
\end{aligned}
$$

This implies (6.2).

$\left.2^{\circ}\right)$ We next claim that $\tilde{\Phi}$ satisfies

$$
\tilde{\Phi}^{\prime}(r)>-C \frac{\sigma\left(r^{1 / 2}\right)^{2}}{r}-C \frac{\sigma\left(r^{1 / 2}\right)}{r} \sqrt{\tilde{\Phi}(r)}, \quad 0<r<r_{\omega} .
$$

To this end, let $\Omega_{ \pm}^{-r^{2}}=\left\{w_{ \pm}\left(\cdot,-r^{2}\right)>0\right\}$. Denoting by $\lambda_{ \pm}=r^{2} \lambda^{-r^{2}}\left(\Omega_{ \pm}^{-r^{2}}\right)$ the normalized eigenvalues of $\Omega_{ \pm}^{-r^{2}}$, as defined in Lemma 2.4, we have

$$
\lambda_{+}+\lambda_{-} \geq 1
$$

Now, by the definition of $\lambda_{ \pm}$, we also have

$$
\lambda_{ \pm} \int_{\Omega_{ \pm}^{-r^{2}}} f^{2} d \gamma^{-r^{2}} \leq r^{2} \int_{\Omega_{ \pm}^{-r^{2}}}|\nabla f|^{2} d \gamma^{-r^{2}}
$$

and therefore, using Proposition 4.1, we obtain that

$$
2\left(1-c_{n} \theta(r)\right) A_{ \pm}(r) \leq C r^{4}+C r^{3} \sqrt{B_{+}(r) / \lambda_{ \pm}}+r^{2} B_{ \pm}(r) / \lambda_{ \pm} .
$$

We may rewrite the previous inequality as

$$
r^{2} \frac{B_{ \pm}(r) / \lambda_{ \pm}}{A_{ \pm}(r)} \geq 2\left(1-c_{n} \theta(r)\right)-\frac{C r^{4}}{A_{ \pm}(r)}-\frac{C r^{3} \sqrt{B_{ \pm}(r) / \lambda_{ \pm}}}{A_{ \pm}(r)} .
$$

Using (6.6), we next obtain estimates on $\tilde{\Phi}^{\prime}(r)$ by considering three possibilities. $\left.2 . i^{\circ}\right) r^{2} B_{+}(r) \geq 2 A_{+}(r)\left(\right.$ or $\left.r^{2} B_{-}(r) \geq 2 A_{-}(r)\right)$. Then from (6.1) we have

$$
\tilde{\Phi}^{\prime}(r)=\frac{\tilde{\Phi}(r)}{r}\left(-4+2 c_{0} \theta(r)+2 r^{2} \frac{B^{+}}{A^{+}}+2 r^{2} \frac{B^{-}}{A^{-}}\right) \geq 0 .
$$


2.ii $i^{\circ} r^{2} B_{+}(r) \leq 2 A_{+}(r)$ and $\lambda_{+} \geq 1\left(\right.$ or $r^{2} B_{-}(r) \leq 2 A_{-}(r)$ and $\left.\lambda_{-} \geq 1\right)$. Then by (6.6) we have

$$
r^{2} \frac{B_{+}}{A_{+}} \geq 2\left(1-c_{n} \theta(r)\right)-\frac{C r^{4}}{A_{+}}-\frac{C r^{2}}{\sqrt{A_{+}}} .
$$

Then, assuming $c_{0}>2 c_{n}$, from (6.1) we obtain

$$
\begin{aligned}
\tilde{\Phi}^{\prime}(r) & =\frac{2 \tilde{\Phi}(r)}{r}\left\{\left[r^{2} \frac{B_{+}}{A_{+}}-\left(2-c_{0} \theta(r)\right)\right]+r^{2} \frac{B_{-}}{A_{-}}\right\} \\
& \geq-\frac{C \tilde{\Phi}(r)}{r}\left[\frac{r^{4}}{A_{+}}+\frac{r^{2}}{\sqrt{A_{+}}}\right] \\
& \geq-\frac{C e^{2 c_{0} g(r)}}{r} A_{-}-\frac{C e^{c_{0} g(r)} \sqrt{\tilde{\Phi}(r)}}{r} \sqrt{A_{-}} \\
& \geq-C \frac{\sigma\left(r^{1 / 2}\right)^{2}}{r}-C \frac{\sigma\left(r^{1 / 2}\right)}{r} \sqrt{\tilde{\Phi}(r)} .
\end{aligned}
$$

2.iii $\left.{ }^{\circ}\right) r^{2} B_{ \pm}(r) \leq 2 A_{ \pm}(r)$ and $\lambda_{ \pm} \leq 1$. Then by (6.6) we have

$$
r^{2} \frac{B_{ \pm}}{A_{ \pm}} \geq 2 \lambda_{ \pm}\left(1-c_{n} \theta(r)\right)-\frac{C r^{4}}{A_{ \pm}}-\frac{C r^{2}}{\sqrt{A_{ \pm}}} .
$$

Using now that $\lambda_{+}+\lambda_{-} \geq 1$ and assuming $c_{0} \geq 2 c_{n}$, from (6.1) we obtain

$$
\begin{aligned}
\tilde{\Phi}^{\prime}(r) & =\frac{2 \tilde{\Phi}(r)}{r}\left\{\left[r^{2} \frac{B_{+}}{A_{+}}-\left(2-c_{0} \theta(r)\right) \lambda_{+}\right]+\left[r^{2} \frac{B_{-}}{A_{-}}-\left(2-c_{0} \theta(r)\right) \lambda_{-}\right]\right\} \\
& \geq-\frac{C \tilde{\Phi}(r)}{r}\left[\frac{r^{4}}{A_{+}}+\frac{r^{2}}{\sqrt{A_{+}}}+\frac{r^{4}}{A_{-}}+\frac{r^{2}}{\sqrt{A_{-}}}\right] \\
& \geq-\frac{C e^{2 c_{0} g(r)}}{r}\left[A_{+}+A_{-}\right]-\frac{C e^{c_{0} g(r)} \sqrt{\tilde{\Phi}(r)}}{r}\left[\sqrt{A_{+}}+\sqrt{A_{-}}\right] \\
& \geq-C \frac{\sigma\left(r^{1 / 2}\right)^{2}}{r}-C \frac{\sigma\left(r^{1 / 2}\right)}{r} \sqrt{\tilde{\Phi}(r)}
\end{aligned}
$$

for $r<r_{\omega}$.

Thus, we see that the inequality (6.3) holds in all cases.

$3^{\circ}$ ) We next claim that

$$
\frac{d}{d r}\left[\left(\tilde{\Phi}(r)+C \sigma_{2}(r)\right)^{1 / 2}+C \sigma_{1}(r)\right] \geq 0, \quad 0<r<r_{\omega}
$$

where

$$
\sigma_{1}(r)=\int_{0}^{r} \frac{\sigma\left(\rho^{1 / 2}\right)}{\rho} d \rho, \quad \sigma_{2}(r)=\int_{0}^{r} \frac{\sigma\left(\rho^{1 / 2}\right)^{2}}{\rho} d \rho .
$$

Indeed, (6.7) is equivalent to

$$
\tilde{\Phi}^{\prime}(r) \geq-C \sigma_{2}^{\prime}(r)-2 \sigma_{1}^{\prime}(r)\left(\tilde{\Phi}(r)+C \sigma_{2}(r)\right)^{1 / 2}
$$

which follows easily from (6.3). 
$\left.4^{\circ}\right)$ Taking now $0<r \leq \rho \leq r_{\omega}$, we obtain

$$
\begin{aligned}
\sqrt{\tilde{\Phi}(r)} & \leq\left(\tilde{\Phi}(r)+C \sigma_{2}(r)\right)^{1 / 2}+C \sigma_{1}(r) \\
& \leq\left(\tilde{\Phi}(\rho)+C \sigma_{2}(\rho)\right)^{1 / 2}+C \sigma_{1}(\rho) \\
& \leq \sqrt{\tilde{\Phi}(\rho)}+C\left[\sqrt{\sigma_{2}(\rho)}+\sigma_{1}(\rho)\right] .
\end{aligned}
$$

Note that

$$
2 \sqrt{\sigma_{2}(r)} \leq 2 \sqrt{\sigma\left(r^{1 / 2}\right) \sigma_{1}(r)} \leq \sigma\left(r^{1 / 2}\right)+\sigma_{1}(r)
$$

and therefore, introducing

$$
\tilde{\sigma}(r):=\sigma\left(r^{1 / 2}\right)+\sigma_{1}(r),
$$

we have

$$
\sqrt{\tilde{\Phi}(r)} \leq \sqrt{\tilde{\Phi}(\rho)}+C \tilde{\sigma}(\rho) .
$$

Squaring and using that $(a+b)^{2} \leq a^{2}[1+\tilde{\sigma}(\rho)]+b^{2}[1+1 / \tilde{\sigma}(\rho)]$ with $a=\sqrt{\tilde{\Phi}(\rho)}$ and $b=C \tilde{\sigma}(\rho)$, we obtain

$$
\tilde{\Phi}(r) \leq[1+\tilde{\sigma}(\rho)] \tilde{\Phi}(\rho)+C \tilde{\sigma}(\rho), \quad 0<r \leq \rho \leq r_{\omega} .
$$

Now recalling that $\tilde{\Phi}(r)=e^{2 c_{0} g(r)} \Phi(r)$ and using that $e^{2 c_{0} g(\rho)} \leq 1+C_{0} g(\rho)$ for $0<\rho \leq r_{\omega}$, provided $r_{\omega}$ is so small that $g\left(r_{\omega}\right)<1$, we arrive at

$$
\Phi(r) \leq\left[1+\left(1+C_{0}\right) \tilde{\sigma}(\rho)+C_{0} g(\rho)\right] \Phi(\rho)+C \tilde{\sigma}(\rho) .
$$

This implies the theorem with $\alpha(\rho)=\left(1+C_{0}\right) \tilde{\sigma}(\rho)+C_{0} g(\rho)$, which clearly has the required form.

\section{An Application}

The almost monotonicity formulas proved in the previous sections can be applied to various free boundary problems, such as the ones considered in [CJK02], with more general assumptions on the governing operator. In this section, however, we give an application of the almost monotonicity formula to a quasilinear obstacle type problem

$$
\begin{aligned}
& \operatorname{div}\left(a\left(|\nabla u|^{2}\right) \nabla u\right)=f(x, u, \nabla u) \chi_{\Omega}, \\
& |\nabla u|=0 \quad \text { on } \Omega^{c}
\end{aligned}
$$

in a certain domain $D$ in $\mathbb{R}^{n}$, where $\Omega$ is an apriori unknown open set. This kind of free boundary problems appear e.g. in mean-field models describing type II superconductors, see [BBC94].

If the operator is uniformly elliptic and $f$ is bounded, then from the general theory of quasilinear equations, (7.1) alone would imply that $u \in C_{\text {loc }}^{1, \beta}(D)$ for some $0<\beta<1$. However, to study the finer regularity properties of the free boundary $\Gamma=\partial \Omega \cap D$, one in fact will need the optimal $C_{\text {loc }}^{1,1}$-regularity of $u$, see [CSS04], where a simplified equation with $a \equiv 1$ is considered. 
We will make the following assumptions on the functions $a: \mathbb{R}_{+} \rightarrow \mathbb{R}$ and $f: D \times \mathbb{R} \times \mathbb{R}^{n} \rightarrow \mathbb{R}:$

$$
\begin{aligned}
& a \in C_{\text {loc }}^{1, \alpha}([0, \infty)), \\
& a(q), a(q)+2 a^{\prime}(q) q \in\left[\lambda_{0}, 1 / \lambda_{0}\right] \quad \text { for any } q \geq 0 \text { with } \lambda_{0}>0, \\
& |f|+\left|\nabla_{x} f\right|+\left|\partial_{z} f\right|+\left|\nabla_{p} f\right| \leq M \quad \text { uniformly for }(x, z, p) \in D \times \mathbb{R} \times \mathbb{R}^{n} .
\end{aligned}
$$

Note that (7.4) is the uniform ellipticity condition on the quasilinear operator $\operatorname{div}\left(a\left(|\nabla u|^{2}\right) \nabla u\right)$, while (7.5) means that $f$ is uniformly bounded and Lipschitz continuous with respect to all its variables.

Theorem 7.1. Let $u \in W^{1,2}\left(B_{1}\right) \cap L^{\infty}\left(B_{1}\right)$ be a weak (distributional) solution of (7.1)-(7.2) with assumptions (7.3)-(7.5). Then $u \in C_{\mathrm{loc}}^{1,1}\left(B_{1}\right)$ and

$$
\|u\|_{C^{1,1}\left(B_{1 / 2}\right)} \leq C\left(C_{a}, \alpha, n, \lambda_{0}, M,\|u\|_{L^{\infty}\left(B_{1}\right)}\right)
$$

with $C_{a}=\|a\|_{C^{1, \alpha}\left(\left[0, R\left(n, \lambda_{0}, M,\|u\|_{\left.L^{\infty}\left(B_{1}\right)\right)}\right]\right)\right.}$.

This theorem generalizes that of Shahgholian [Sha03] for equations of the type $\Delta u=f(x, u) \chi_{\Omega},|\nabla u|=0$ on $\Omega^{c}$. See also the work of Uraltseva [Ura01] for a similar result in a two-phase membrane problem. We explicitly remark here that the result in Theorem 7.1 is new even in the case $a \equiv 1$ when $f(x, u, \nabla u)$ depends nontrivially on $x, u, \nabla u$.

The key idea and the connection with the almost monotonicity formulas is seen in the following lemma.

Lemma 7.2. Let $u$ be as in Theorem 7.1. Then for any direction e the functions $w_{ \pm}=\left(\partial_{e} u\right)^{ \pm}=\max \left\{ \pm \partial_{e} u, 0\right\}$ satisfy

$$
w_{ \pm} \geq 0, \quad \operatorname{div}\left(\mathcal{A}(x) \nabla w_{ \pm}\right)+b(x) \nabla w_{ \pm}+c(x) w_{ \pm} \geq-M, \quad w_{+} \cdot w_{-}=0,
$$

where

$$
\begin{aligned}
\mathcal{A}(x) & =a\left(|\nabla u(x)|^{2}\right) I+2 a^{\prime}\left(|\nabla u(x)|^{2}\right) \nabla u(x) \otimes \nabla u(x), \\
b(x) & =-\left(\nabla_{p} f\right)(x, u(x), \nabla u(x)), \\
c(x) & =-\left(\partial_{z} f\right)(x, u(x), \nabla u(x)) .
\end{aligned}
$$

Remark. Note that from equation (7.1) we have $u \in C_{\text {loc }}^{1, \beta}\left(B_{1}\right)$ and therefore $\mathcal{A} \in$ $C_{\mathrm{loc}}^{\alpha \beta}\left(B_{1}\right)$, so the double Dini continuity condition on $\mathcal{A}$ is satisfied. Also, the condition (7.5) implies the uniform boundedness of $b$ and $c$. Furthermore, since the exponent $\beta$ and $\|u\|_{C^{1, \beta}\left(B_{1 / 2}\right)}$ depend only on $n, \lambda_{0}, M$ and $\|u\|_{L^{\infty}\left(B_{1}\right)}$, the structural constants in (1.2)-(1.5) depend only on the latter constants and the $C^{1, \alpha}$ norm of $a$ on $\left[0,\|\nabla u\|_{L^{\infty}\left(B_{3 / 4}\right)}^{2}\right]$.

Proof of Lemma 7.2. The idea of the proof is as follows: in the open set $\Omega_{e}^{+}=$ $\left\{\partial_{e} u>0\right\} \subset \Omega$ we may differentiate the equation to obtain

$$
\begin{array}{r}
\partial_{e} \operatorname{div}\left(a\left(|\nabla u|^{2}\right) \nabla u\right)=\operatorname{div}\left[a\left(|\nabla u|^{2}\right) \nabla\left(\partial_{e} u\right)+2 a^{\prime}\left(|\nabla u|^{2}\right) \nabla u \nabla\left(\partial_{e} u\right) \nabla u\right] \\
=e \nabla_{x} f+\partial_{e} u \partial_{z} f+\nabla\left(\partial_{e} u\right) \nabla_{p} f,
\end{array}
$$

which implies that

$$
\mathcal{L}_{\mathcal{A}, b, c}\left(\partial_{e} u\right)=e \nabla_{x} f \geq-M \quad \text { in } \Omega_{e}^{+} .
$$


Then by using Kato's inequality [Kat72], we conclude that

$$
\mathcal{L}_{\mathcal{A}, b, c}\left(\partial_{e} u\right)^{+} \geq-M \text { in } D .
$$

In fact, (7.6)-(7.7) is quite easy to justify in the sense of distributions and we therefore have for $w=\partial_{e} u$

$$
\int_{D}-\mathcal{A}(x) \nabla w \nabla \eta+b(x) \nabla w \eta+c(x) w \eta \geq-M \int_{D} \eta
$$

for any $\eta \in W_{0}^{1,2}\left(\Omega_{e}^{+}\right), \eta \geq 0$. To justify (7.8), we choose

$$
\eta=\chi\left(\frac{w}{\varepsilon}\right) \psi(x)
$$

where $\psi \in C_{0}^{\infty}(D), \psi \geq 0$, and $\chi \in C^{\infty}(\mathbb{R})$ is such that

$$
\chi^{\prime}(t) \geq 0, \quad \chi(t)=0 \text { for } t \leq 1, \quad \chi(t)=1 \text { for } t \geq 2 .
$$

Then we have

$$
\begin{array}{r}
\int_{D}-[\mathcal{A}(x) \nabla w \nabla w] \frac{1}{\varepsilon} \chi^{\prime}\left(\frac{w}{\varepsilon}\right) \psi+\chi\left(\frac{w}{\varepsilon}\right)[-\mathcal{A}(x) \nabla w \nabla \psi+b(x) \nabla w \psi+c(x) w \psi] \\
\geq-M \int_{D} \chi\left(\frac{w}{\varepsilon}\right) \psi \geq-M \int_{D} \psi .
\end{array}
$$

Now using that $\mathcal{A}(x) \nabla w \nabla w \geq 0, \chi^{\prime} \geq 0, \psi \geq 0$, we may throw away the first term in the above integral to obtain

$$
\int_{D} \chi\left(\frac{w}{\varepsilon}\right)[-\mathcal{A}(x) \nabla w \nabla \psi+b(x) \nabla w \psi+c(x) w \psi] \geq-M \int_{D} \psi .
$$

Noticing that $\chi(w / \varepsilon) \rightarrow \chi_{\Omega_{e}^{+}}$a.e. as $\varepsilon \rightarrow 0+$ and using the dominated convergence theorem, we obtain that

$$
\int_{\Omega_{e}^{+}}-\mathcal{A}(x) \nabla w \nabla \psi+b(x) \nabla w \psi+c(x) w \psi \geq-M \int_{D} \psi,
$$

which is equivalent to $\mathcal{L}_{\mathcal{A}, b, c} w^{+} \geq-M$. The proof of the lemma is complete.

Proof of Theorem 7.1. First, without loss of generality, we assume that $\|u\|_{L^{\infty}\left(B_{1}\right)} \leq$ 1 , otherwise we replace $u$ with $u /\left(1+\|u\|_{L^{\infty}\left(B_{1}\right)}\right)$, which satisfies an equation of the type (7.1) with rescaled $a$ and $f$.

Next, from the Calderón-Zygmund estimates it follows that $u \in W_{\mathrm{loc}}^{2, p}\left(B_{1}\right)$ for any $1<p<\infty$. Fixing a $p>n$, this implies that at any Lebesgue point $x_{0}$ of $D^{2} u$ the function $u$ is twice differentiable, see e.g. [Eva98, Theorem 5.8.5]. Then we fix such $x_{0} \in B_{1 / 2}$ and define

$$
w(x)=\partial_{e} u(x)
$$

for a unit vector $e$ orthogonal to $\nabla u\left(x_{0}\right)$ (if $\nabla u\left(x_{0}\right)=0$, take arbitrary unit $e$ ). Without loss of generality we may assume $x_{0}=0$. Our aim is to obtain a uniform estimate for $\partial_{x_{j}} e u(0)=\partial_{x_{j}} w(0), j=1, \ldots, n$. By construction, $w(0)=0$ and $w$ is differentiable at 0 . Hence, we have the Taylor expansion

$$
w(x)=\xi \cdot x+o(|x|), \quad \xi=\nabla w(0) .
$$

Now, if $\xi=0$ then $\partial_{x_{j}} w(0)=0$ for all $j=1, \ldots, n$ and there is nothing to estimate. If $\xi \neq 0$, consider the cone

$$
\mathcal{C}_{\xi}=\left\{x \in \mathbb{R}^{n}: \xi \cdot x \geq|\xi||x| / 2\right\},
$$


which has a property that

$$
\mathcal{C}_{\xi} \cap B_{r} \subset\{w>0\}, \quad-\mathcal{C}_{\xi} \cap B_{r} \subset\{w<0\}
$$

for sufficiently small $r>0$. Consider also the rescalings

$$
w_{r}(x)=\frac{w(r x)}{r}, \quad x \in B_{1} .
$$

Note that $w_{r}(x) \rightarrow w_{0}(x):=\xi \cdot x$ uniformly in $B_{1}$ and $\nabla w_{r} \rightarrow \nabla w_{0}$ in $L^{p}\left(B_{1}\right)$, $p>n$. The latter follows from the equality

$$
\int_{B_{1}}\left|\nabla w_{r}(x)-\xi\right|^{p} d x=\frac{1}{r^{n}} \int_{B_{r}}|\nabla w(x)-\nabla w(0)|^{p} d x,
$$

where the right-hand side goes to zero as $r \rightarrow 0$, since $x_{0}=0$ is a Lebesgue point for $\nabla w$. Then for $c_{n}>0$ we have

$$
\begin{aligned}
c_{n}|\xi|^{4} & =\int_{\mathcal{C}_{\xi} \cap B_{1}} \frac{\left|\nabla w_{0}(x)\right|^{2} d x}{|x|^{n-2}} \int_{-\mathcal{C}_{\xi} \cap B_{1}} \frac{\left|\nabla w_{0}(x)\right|^{2} d x}{|x|^{n-2}} \\
& =\lim _{r \rightarrow 0} \int_{\mathcal{C}_{\xi} \cap B_{1}} \frac{\left|\nabla w_{r}(x)\right|^{2} d x}{|x|^{n-2}} \int_{-\mathcal{C}_{\xi} \cap B_{1}} \frac{\left|\nabla w_{r}(x)\right|^{2} d x}{|x|^{n-2}} \\
& =\lim _{r \rightarrow 0} \frac{1}{r^{4}} \int_{\mathcal{C}_{\xi} \cap B_{r}} \frac{|\nabla w(x)|^{2} d x}{|x|^{n-2}} \int_{-\mathcal{C}_{\xi} \cap B_{r}} \frac{|\nabla w(x)|^{2} d x}{|x|^{n-2}} \\
& \leq \liminf _{r \rightarrow 0} \frac{1}{r^{4}} \int_{B_{r}} \frac{\left|\nabla w^{+}(x)\right|^{2} d x}{|x|^{n-2}} \int_{B_{r}} \frac{\left|\nabla w^{-}(x)\right|^{2} d x}{|x|^{n-2}},
\end{aligned}
$$

where in the last step we have use the inclusion $\pm \mathcal{C}_{\xi} \cap B_{r} \subset\{ \pm w>0\}$ for small $r>0$. Summarizing, we obtain that

$$
|\nabla w(0)|^{4} \leq C_{n} \liminf _{r \rightarrow 0} \varphi\left(r, w^{+}, w^{-}\right),
$$

where $\varphi$ is as in Theorem III. Now, by Lemma $7.2, w^{ \pm}$satisfy the conditions of Theorem III (in $B_{1 / 2}$ instead of $B_{1}$ ) and therefore we have

$$
\liminf _{r \rightarrow 0} \varphi\left(r, w^{+}, w^{-}\right) \leq C\left(1+\|w\|_{L^{2}\left(B_{1 / 2}\right)}^{2}\right)^{2} \leq C .
$$

(Recall that we assume $\|u\|_{L^{\infty}\left(B_{1}\right)} \leq 1$ which gives that $\|w\|_{L^{2}\left(B_{1 / 2}\right)} \leq C$.) The latter implies that

$$
\left|\nabla \partial_{e} u\left(x_{0}\right)\right| \leq C
$$

which doesn't yet give the desired estimate on $\left|D^{2} u\left(x_{0}\right)\right|$, since $e$ is subject to the condition $e \cdot \nabla u\left(x_{0}\right)=0$, unless $\nabla u\left(x_{0}\right)=0$. If $\nabla u\left(x_{0}\right) \neq 0$, we may choose the coordinate system so that $\nabla u\left(x_{0}\right)$ is parallel to $e_{1}$. Then, taking $e=e_{2}, \ldots, e_{n}$ in the estimate above, we obtain

$$
\left|\partial_{x_{i} x_{j}} u\left(x_{0}\right)\right| \leq C, \quad i=2, \ldots, n, \quad j=1,2, \ldots, n .
$$

To obtain the estimate in the missing direction $e_{1}$, we notice that since $u \in$ $W_{\text {loc }}^{2, p}\left(B_{1}\right)$, the equation $\operatorname{div}\left(a\left(|\nabla u|^{2}\right) \nabla u\right)=f(x, u, \nabla u)$ is also satisfied in the strong sense

$$
\left[a\left(|\nabla u|^{2}\right) \delta_{i j}+2 a^{\prime}\left(|\nabla u|^{2}\right) \partial_{x_{i}} u \partial_{x_{j}} u\right] \partial_{x_{i} x_{j}} u+b(x) \nabla u+c(x) u=g(x),
$$

where

$$
g(x)=f(x, u(x), \nabla u(x)) \chi_{\Omega}(x) .
$$


In particular, we may assume that this equation is satisfied at $x_{0}$. Then at $x_{0}$ the second-order term has the form

$$
a\left(\left|\nabla u\left(x_{0}\right)\right|^{2}\right) \Delta u\left(x_{0}\right)+2 a^{\prime}\left(\left|\nabla u\left(x_{0}\right)\right|^{2}\right)\left|\nabla u\left(x_{0}\right)\right|^{2} \partial_{x_{1} x_{1}} u\left(x_{0}\right)
$$

and thus using (7.4) we obtain

$$
\lambda_{0}\left|\partial_{x_{1} x_{1}} u\left(x_{0}\right)\right| \leq\left|g\left(x_{0}\right)\right|+\frac{1}{\lambda_{0}} \sum_{k=2}^{n}\left|\partial_{x_{k} x_{k}} u\left(x_{0}\right)\right|+M\left|\nabla u\left(x_{0}\right)\right|+M\left|u\left(x_{0}\right)\right| .
$$

This implies the missing estimate

$$
\left|\partial_{x_{1} x_{1}} u\left(x_{0}\right)\right| \leq C
$$

and completes the proof of the theorem.

\section{REFERENCES}

[ACF84] Hans Wilhelm Alt, Luis A. Caffarelli, and Avner Friedman, Variational problems with two phases and their free boundaries, Trans. Amer. Math. Soc. 282 (1984), no. 2, 431461. MR732100 (85h:49014)

[Bec89] William Beckner, A generalized Poincaré inequality for Gaussian measures, Proc. Amer. Math. Soc. 105 (1989), no. 2, 397-400. MR954373 (89m:42027)

[BKP98] W. Beckner, C. Kenig, and J. Pipher, A convexity property of eigenvalues with applications, 1998, unpublished.

[BBC94] H. Berestycki, A. Bonnet, and S. J. Chapman, A semi-elliptic system arising in the theory of type-II superconductivity, Comm. Appl. Nonlinear Anal. 1 (1994), no. 3, 1-21. MR1295490 (95e:35192)

[Caf93] Luis A. Caffarelli, A monotonicity formula for heat functions in disjoint domains, Boundary value problems for partial differential equations and applications, RMA Res. Notes Appl. Math., vol. 29, Masson, Paris, 1993, pp. 53-60. MR1260438 (95e:35096)

[CK98] Luis A. Caffarelli and Carlos E. Kenig, Gradient estimates for variable coefficient parabolic equations and singular perturbation problems, Amer. J. Math. 120 (1998), no. 2, 391-439. MR1613650 (99b:35081)

[CJK02] Luis A. Caffarelli, David Jerison, and Carlos E. Kenig, Some new monotonicity theorems with applications to free boundary problems, Ann. of Math. (2) 155 (2002), no. 2, 369404. MR1906591 (2003f:35068)

[CSS04] Luis Caffarelli, Jorge Salazar, and Henrik Shahgholian, Free-boundary regularity for a problem arising in superconductivity, Arch. Ration. Mech. Anal. 171 (2004), no. 1, 115128. MR2029533 (2004m:82156)

[EP08] Anders Edquist and Arshak Petrosyan, A parabolic almost monotonicity formula, Math. Ann. 341 (2008), no. 2, 429-454. MR2385663

[Eva98] Lawrence C. Evans, Partial differential equations, Graduate Studies in Mathematics, vol. 19, American Mathematical Society, Providence, RI, 1998. MR1625845 (99e:35001)

[FH76] S. Friedland and W. K. Hayman, Eigenvalue inequalities for the Dirichlet problem on spheres and the growth of subharmonic functions, Comment. Math. Helv. 51 (1976), no. 2, 133-161. MR0412442 (54 \#568)

[Kat72] Tosio Kato, Schrödinger operators with singular potentials, Proceedings of the International Symposium on Partial Differential Equations and the Geometry of Normed Linear Spaces (Jerusalem, 1972), 1972, pp. 135-148 (1973). MR0333833 (48 \#12155)

[Gro75] Leonard Gross, Logarithmic Sobolev inequalities, Amer. J. Math. 97 (1975), no. 4, 10611083. MR0420249 (54 \#8263)

[LSU68] O. A. Ladyženskaja, V. A. Solonnikov, and N. N. Ural'ceva, Lineinye i kvazilineinye uravneniya parabolicheskogo tipa, Izdat. "Nauka", Moscow, 1968 (Russian); English transl., Linear and quasilinear equations of parabolic type, Translations of Mathematical Monographs, Vol. 23, American Mathematical Society, Providence, R.I., 1967. MR0241822 (39 \#3159b). MR0241821 (39 \#3159a)

[Sha03] Henrik Shahgholian, $C^{1,1}$ regularity in semilinear elliptic problems, Comm. Pure Appl. Math. 56 (2003), no. 2, 278-281. MR1934623 (2003h:35087) 
[Ura01] N. N. Uraltseva, Two-phase obstacle problem, J. Math. Sci. (New York) 106 (2001), no. 3, 3073-3077. Function theory and phase transitions. MR1906034 (2003e:35331)

Department of Applied Mathematics and Theoretical Physics, University of CamBRIDGe, CAMBridge CB3 0WA, UK

E-mail address: n.matevosyan@damtp.cam.ac.uk

Department of Mathematics, Purdue University, West Lafayette, in 47907, USA

E-mail address: arshak@math.purdue.edu 